\title{
Nuclear Spin Selective Torsional States: Implications of Molecular Symmetry
}

\author{
By Steffen Belz ${ }^{1}$, Omar Deeb ${ }^{2}$, Leticia González ${ }^{3}$, Thomas Grohmann ${ }^{1, *}$, \\ Daniel Kinzel $^{3}$, Monika Leibscher ${ }^{1,4}$, Jörn Manz ${ }^{1,5}$, Rana Obaid ${ }^{2,3, *}$, \\ Markus Oppel $^{3, *}$, George Densingh Xavier ${ }^{6,7}$, and Shmuel Zilberg ${ }^{6}$ \\ ${ }^{1}$ Institut für Chemie and Biochemie, Freie Universität Berlin, Takustraße 3, 14195 Berlin, Germany \\ ${ }^{2}$ Faculty of Pharmacy, Al-Quds University, Abu Dis, Palestine \\ ${ }^{3}$ Institut für Theoretische Chemie, Universität Wien, Währinger Straße 17, 1090 Vienna, Austria \\ ${ }^{4}$ Institut für Theoretische Physik, Leibniz Universität Hannover, Appelstraße 2, 30167 Hannover, \\ Germany \\ ${ }^{5}$ Laser Spectroscopy Laboratory, Shanxi University, Wucheng Road 92, 030006 Taiyuan, China \\ ${ }^{6}$ Institute of Chemistry, The Edmond Safra Campus, Givat Ram, The Hebrew University of Jerusalem, \\ 91904 Jerusalem, Israel \\ ${ }^{7}$ Department of Chemistry, Indian Institute of Technology, 600032 Madras, India
}

This paper is dedicated to Prof. Hans-Heinrich Limbach on the occasion of his 70th birthday, in gratitude for his continuous support of, and stimulating interest in our trilateral research project.

(Received January 4, 2013; accepted in revised form March 5, 2013)

(Published online April 22, 2013)

\section{Nuclear Spin Isomers / Molecular Torsion / Molecular Rotors / Molecular Symmetry / Group Theory / Quantum Chemistry / Symmetry-Adapted Quantum Dynamics}

We consider a class of molecules with $\mathcal{C}_{2}$ symmetry axis and three segments $\mathrm{A}, \mathrm{B}, \mathrm{C}$ which can rotate independently about that axis, corresponding to two independent torsions (B vs. A and $\mathrm{C}$ vs. B). The torsions may be feasible either in the electronic ground or in the excited states. We determine the corresponding molecular symmetry group, i.e. the Abelian group $\mathrm{G}_{16}^{\mathrm{A}}$ representing 16 feasible permutations and permutation-inversions, and its permutation subgroup with eight permutations, together with their properties, e.g. their character tables and the corresponding 16 or 8 irreducible representations (IREPs), respectively. Accordingly, the molecules which belong to this class have at most eight different nuclear spin isomers (NSIs). A subset of them "survives" at low temperature, $T \rightarrow 0$. The corresponding NSI selective wavefunctions contain products of torsional times nuclear wavefunctions with specific IREPs. The NSIs are characterized by these IREPs. As an example, we determine the molecular symmetry adapted torsional wavefunctions of the model 2-[4(cyclopenta-2,4-dien-1-ylidene)cyclohexa-2,5-dien-1-ylidene]-2H-1,3-dioxole, abbreviated as CCD. In order to demonstrate the principles of the derivations, we employ a simple model, with the $\mathcal{C}_{2}$ symmetry axis oriented along the laboratory $Z$-axis, and with all degrees of freedom frozen

* Corresponding authors. E-mail: thomas.grohmann@fu-berlin.de; rana.obaid@univie.ac.at; markus.oppel@univie.ac.at 
in the equilibrium structure of $\mathrm{CCD}$, except the two torsional degrees of freedom. The resulting torsional wavefunctions represent different NSIs of CCD, ready for subsequent applications, e.g. for demonstrations of NSI selective dynamics.

\section{Introduction}

This paper aims at extending previous quantum mechanical studies of NSIs, from established systems to a new class of molecules. This task will call for the appropriate, new molecular symmetry group, with applications to nuclear spin isomer selective molecular properties. The following introduction guides the reader to the relevant topics, from important historical aspects that serve as a reference, up to the state of the art.

The concept of NSIs was introduced independently by Heisenberg [1] and Hund [2]. As an example, they considered molecular hydrogen and postulated that $\mathrm{H}_{2}$ should exist in the form of two NSIs, para-hydrogen $\left(\mathrm{p}-\mathrm{H}_{2}\right)$ and ortho-hydrogen $\left(\mathrm{o}-\mathrm{H}_{2}\right)$. The corresponding nuclear spin wavefunctions are ungerade and gerade with respect to permutations of the two protons, respectively. These nuclear spin wavefunctions are multiplied by rotational wavefunctions with gerade (even rotational quantum numbers $J$ ) and ungerade (odd values of $J$ ) parities, respectively. This way, Heisenberg and Hund warranted antisymmetry of the total wavefunction with respect to the exchange of the two fermionic nuclei.

The ansatz of Heisenberg and Hund implies enormous consequences: Essentially, different NSIs have different properties, hence they appear as different species, at least on time scales shorter than nuclear spin conversion. For example, the rotational energies of $\mathrm{o}-\mathrm{H}_{2}$ and $\mathrm{p}-\mathrm{H}_{2}$ are different, thus they have different temperature dependencies of their concentrations or populations, of the internal energies and, as a consequence, of the heat capacities [3,4]. The experimental verification of this prediction by Bonhoeffer and Harteck [5] marks one of the great successes of quantum theory. At the same time, the quantum theory of Heisenberg and Hund could explain the anomalous specific heat capacity of normal hydrogen $\left(n-\mathrm{H}_{2}\right)$, first measured by Eucken [6].

Another success of their theory came from extended applications to various homonuclear diatomic molecules, explaining the intensity alternations in the rotational spectra, which had first been reported by Mecke [7]. Subsequently, nuclear spin selective spectra were predicted and analyzed for small, rigid polyatomic molecules such as $\mathrm{CH}_{4}, \mathrm{C}_{2} \mathrm{H}_{4}, \mathrm{H}_{2} \mathrm{CO}, \mathrm{H}_{2} \mathrm{O}$ and various isotopomers, and finally also for non-rigid ones, that is for molecules which perform large amplitude motions such as inversion of $\mathrm{NH}_{3}$; for a recent survey, see Ref. [8].

On the theoretical side, the historic success of Heisenberg, Hund, Bonhoeffer and Harteck supported the symmetrization postulate for identical and therefore indistinguishable particles [8], one of the keys to successful quantum evaluations of chemical properties $[3,4]$.

Today, NSI selectivity is an important topic in broad areas of chemistry and physics, including not only the previous phenomena but also many new ones, $e$.g. selective reaction rates [9], boiling or melting points $[8,10]$, heats of vaporization $[8,10]$, or nuclear magnetic resonance (NMR) spectra [11-28].

As a consequence, selective NSIs may allow new, specific applications. For example, NMR signals for molecules which have been prepared by means of pure $\mathrm{p}-\mathrm{H}_{2}$ 
may be enhanced by four to five orders of magnitude, compared to $n-\mathrm{H}_{2}$ - this effect allowed the development of the method with acronym PASADENA [29,30], which was later also called "para-hydrogen induced polarization" (PHIP) [31]. This method allowed the detection of reaction intermediates and the verification of reaction mechanisms, see Refs. [11,23,32,33] and the reviews [34-38], and is a powerful tool for analysis of catalysis [23], in fact even regio- or enantio-selective catalysis [37], and for the characterization of organometallic dihydrides [12-16,39]. PHIP may also be used as a test of reversible hydrogenation [40,41], which, in turn, may support imaging for purposes of diagnoses in medicine [42]. Other applications are based on determinations of the temperature-dependent $\mathrm{o}-\mathrm{H}_{2} / \mathrm{p}-\mathrm{H}_{2}$ nuclear spin conversion rates $[10$, $15,16,19,20,28]$; the resulting ratios of the densities of the NSIs may then serve as a scale for the nuclear spin temperatures, even in remote objects such as Halley's comet $[19,43]$.

All the fundamental properties, analyses and applications of NSIs which have been listed so far are concerned with time-independent or quasi-static properties (that means that they are robust on the relevant time scale for observation), e.g. thermodynamic functions, spectra, nuclear spin conversion rates, and rate coefficients of chemical reactions. The latter ones imply nuclear spin selective time dependent rate processes with new applications, e.g. analyses of specific reaction pathways, even in the time domain [25], e.g. keto-enol tautomerism [26]. Those rate processes enable NSI selective chemical kinetics. They proceed incoherently, on time scales which are much longer, by orders of magnitude, than coherent processes such as molecular rotations, vibrations, or the formation of new chemical bonds during chemical reactions.

The most recent general breakthrough in the field is the discovery of nuclear spin selective coherent quantum dynamics, again starting from applications to diatomic molecules [44-46] via rather small, rigid polyatomic molecules [47-49] to non-rigid ones [49-55]. The results are spectacular: Different NSIs of diatomic or rigid polyatomic molecules show different transient alignment after excitation with moderate intense femtosecond laser pulses, opening new avenues to laser separations of nuclear spin isomers [44-49,55]. Moreover, NSIs of non-rigid polyatomic molecules may have different periods of torsion in electronically excited states [50]; they show different radiation-less decay through conical intersections after photo-excitation [51-54]; and they could be used to realize NSI selective molecular rotors $[46,49,55]$. Previous quantum dynamical investigations of torsional motions suggest various additional applications of NSI selectivity, see e.g. Refs. [56-73].

We are now ready to specify the goal of the present paper: the extension of previous quantum mechanical investigations of NSIs, from non-rigid polyatomic molecules with a single torsional degree of freedom (dof) to molecules with two torsional dof. At the same time, the present work should lay the foundation for an extension of earlier quantum dynamics simulations of nuclear spin selective torsions after photoexcitation [50-54] to systems with two torsional dof. Ultimately, we aim at NSI selective control of these molecular torsions. This goal is motivated by previous demonstrations of quantum control of molecular torsions for various purposes, e.g. energy transfer [77]; enhancement of transient absorption spectroscopy [73]; the ignition of molecular motors $[55,64,78]$; and the preparation of pure enantiomers out of racemic mixtures $[59,61,63,65,73,79]$. 
Specifically, we shall investigate NSI selectivity of molecules which are characterized by two criteria:

(i) Their structures at the minima of the potential energy surface of the electronic ground state ("minimum structures") have a $\mathcal{C}_{2}$ symmetry axis.

(ii) The molecules have three non-identical "torsional" segments which can rotate independently about that $\mathcal{C}_{2}$-axis, possibly in the electronic ground state or in electronic excited states. Those three rotations correspond to two torsional degrees of freedom for the torsions of two neighboring segments with respect to each other, plus the overall rotation of the entire molecule about the $\mathcal{C}_{2}$-axis.

Rigorous evaluations of the quantum dynamics of molecules which belong to this class, in full dimensionality (full-d) are all technically too demanding. To simplify this task, we will assume that the $\mathcal{C}_{2}$ symmetry axis has been pre-oriented, e.g. parallel to the laboratory fixed $Z$-axis. Pioneering experimental [80-82] and theoretical [47,83-91] work suggests that this scenario may be prepared by means of laser pulses; for reviews, see Refs. $[92,93]$. In fact, the first successful experimental and theoretical demonstrations of sequential molecular orientation and ignition of molecular torsions have just been published in Refs. [69] and [55,72,73,94], respectively, culminating in the joint experimental and theoretical work [70].

Furthermore, this exploratory investigation shall employ a simple two-dimensional (2d) model, focusing on the two torsional degrees of freedom. Corresponding lowdimensional (low-d) quantum dynamics simulations of torsional motions in oriented molecules with just two torsional segments have already been presented in Refs. [4973], for example.

The approach will be demonstrated for a model system, specifically the quinodimethane derivative 2-[4-(cyclopenta-2,4-dien-1-ylidene)cyclohexa-2,5-dien-1ylidene]-2H-1,3-dioxole, abbreviated as CCD. One of several equivalent minimum structures (see below) is shown in Fig. 1. For simplicity, the carbon and oxygen nuclei are those of the isotopes ${ }^{12} \mathrm{C}$ and ${ }^{16} \mathrm{O}$, i.e. the NSIs of CCD depend exclusively on the spins of ten protons. The figure legend contains important information about the nomenclature for the three torsional segments labeled A, B, C. Rotations about $180^{\circ}$ of these segments about the $\mathcal{C}_{2}$ symmetry axis interchange equivalent nuclei of fragments (1) and (2), (3) and (4) and (5) and (6), respectively. Another set labeled (0) contains the nuclei on the $\mathcal{C}_{2}$ symmetry axis.

Additional molecules belonging to the present types of systems are shown in Fig. 2. System (I), 1,1-difluoro-quinodimethane, is another representative of the important quinodimethane derivates - this group of molecules has attracted much attention because of their diradical properties [74]. Systems (II)-(V) are important representatives of molecules exhibiting twist-induced charge transfer (TICT), giving rise to dual fluorescence [75]. System (VI) is a promising building block for molecular rotors driven by torsions about the molecular $\mathcal{C}_{2}$-axis [76].

Our investigations of the NSI selectivity of $\mathrm{CCD}$, as representative of the present class of molecules, will proceed in two steps: First, we shall identify the NSIs of CCD in the electronic ground state. At this stage, we shall show that - in the limit of the present $2 \mathrm{~d}$ model - the NSIs may be identified by different torsional states. This step recalls the pioneering work of Heisenberg [1] and Hund [2], who had shown that the NSIs of 


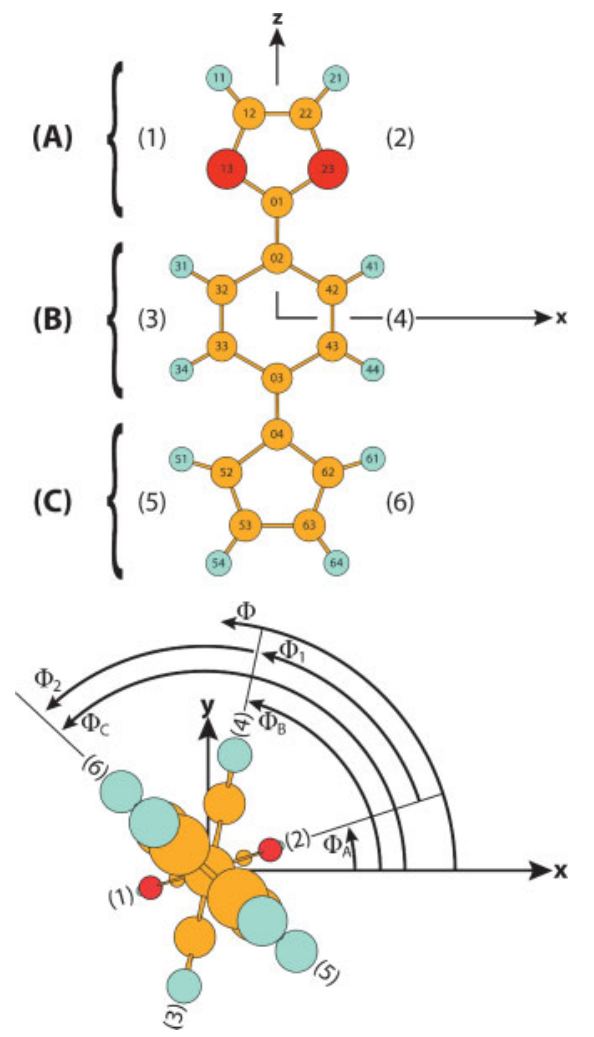

Fig. 1. (Top) Side view of the structure of the quinodimethane derivative 2-[4-(cyclopenta-2,4-dien-1ylidene)cyclohexa-2,5-dien-1-ylidene]-2H-1,3-dioxole (CCD) at one of the four equivalent minima of the potential energy surface for the electronic ground state ("minimum structure"). The local symmetry is $\mathrm{C}_{2 \mathrm{v}}$, with the $\mathcal{C}_{2}$ symmetry axis oriented along the laboratory-fixed $Z$-axis, and with the planar molecule in the $X / Z$ plane. The molecule has three torsional segments labeled (A), (B) and (C) with equivalent fragments (1) and (2), (3) and (4), and (5) and (6), respectively. The set of nuclei which are located on the $\mathcal{C}_{2}$ symmetry axis is called fragment (0). The nuclei are labeled by double indices $i k$ where $i$ denotes the fragment, and $k$ specifies the nucleus within the fragment, e.g. fragments (1) and (2) have hydrogen (light blue), carbon (orange) and oxygen (red) nuclei labeled 11, 12, 13 and 21, 22, 23, with the same labels $k=1,2,3$ for equivalent nuclei in these fragments. (Bottom) top view of CCD after intramolecular rotations of the segments (A), (B), (C) by angles $\Phi_{\mathrm{A}}, \Phi_{\mathrm{B}}, \Phi_{\mathrm{C}}$, respectively, about the symmetry axis $\mathcal{C}_{2}$ (schematic). Also shown are the corresponding torsional angles $\Phi_{1}=\Phi_{\mathrm{B}}-\Phi_{\mathrm{A}}$ and $\Phi_{2}=\Phi_{\mathrm{C}}-\Phi_{\mathrm{B}}$ describing torsions of the neighboring segments (A) vs. (B) and (B) vs. (C), respectively, as well as the angle of overall rotation, $\Phi$. Rotations of the segments (A), (B) or (C) by $\Phi_{A}=180^{\circ}$ degrees, $\Phi_{\mathrm{B}}=180^{\circ}$ or $\Phi_{\mathrm{C}}=180^{\circ}$ about the $\mathcal{C}_{2}$ symmetry axis exchange the equivalent fragments (1) and (2), (3) and (4) or (5) and (6), respectively. Nuclei with the same labels $k$ in fragments (1) and (2), (3) and (4), or (5) and (6) are exchanged upon these rotations, respectively.

$\mathrm{H}_{2}$ are associated with different rotational states - this analogy is valid irrespective of the fact that Heisenberg and Hund had to deal with the exchange of just two protons, whereas the present torsions of CCD involve exchanges of ten protons. Thus for $\mathrm{H}_{2}$, there are $2^{2}=4$ nuclear spin wavefunctions which can be separated into the familiar two sets with 1 and 3 members which can be assigned to $\mathrm{p}-\mathrm{H}_{2}$ and to $\mathrm{o}-\mathrm{H}_{2}$, respectively. 
(I)

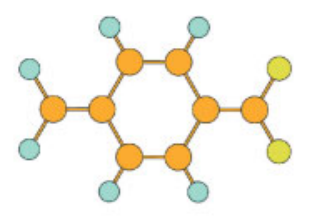

(II)

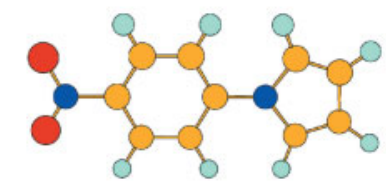

(III)

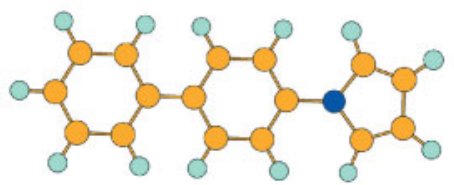

(IV)

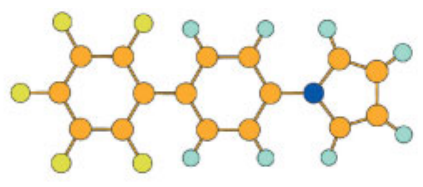

(V)

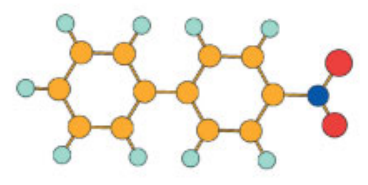

(VI)

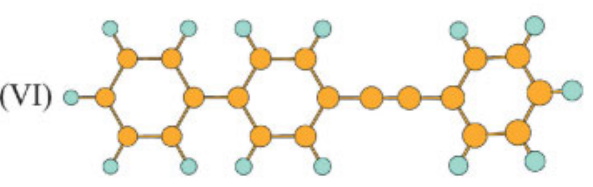

Fig. 2. Molecules with $\mathcal{C}_{2}$ symmetry axis and three segments that can rotate about that axis, see also the criteria (i),(ii): (I) 1,1-difluoro-quinodimethane, (II) 1-pyrrolyl-4-nitro-benzene, (III) 1-pyrrolyl-4-phenylbenzene, (IV) 1-pyrrolyl-4-(per-fluoro-phenyl)-benzene, (V) para-nitro-biphenyl, (VI) para-phenyl-tolane. The color code is: light blue for hydrogen, orange for carbon, yellow for fluorine, dark blue for nitrogen, red for oxygen.

In contrast, CCD supports $2^{10}=1024$ nuclear spin functions - one of the challenges of this paper is to determine how these functions should be separated into corresponding sets for NSIs, and even more fundamentally to discover the number and the characteristics of those NSIs. Another problem that we shall solve is suggested by the well-known fact that $\mathrm{p}-\mathrm{H}_{2}$ is the only NSI which "survives" as the temperature approaches the limit $T \rightarrow 0[3,4]$. By analogy, we shall determine the subset of NSIs of CCD which "survive" as $T \rightarrow 0$. Obviously, these tasks are much more demanding for oriented CCD than for $\mathrm{H}_{2}$, calling for new tools (see the next Paragraph). Suffice it here to say that the present torsional wavefunctions will be evaluated for the potential energy surface (PES) of the model CCD in the electronic ground state; the PES in turn will be calculated by means of ab initio quantum chemistry methods. This first step is the topic of the present paper. It is carried out, however, with the second step already in mind, i.e. to demonstrate that electronic excitations of different NSIs may induce different time evolutions of the torsional wavepacket dynamics in electronic excited states. In fact, this effect will be demonstrated even for the case where there is no torsional dynamics in the electronic ground state, due to an insuperable barrier of the PES. The general scope of that second step is to show that selective laser excitations of different NSIs may yield selective torsional dynamics and, ultimately, selective photochemistry. This will be the topic of a sequel paper.

In order to determine the NSI selective torsional wavefunctions of the present class of molecules, and to classify their symmetries, we shall employ a symmetry concept which is based on those permutations of identical nuclei which may be achieved by feasible torsions. The ubiquitous molecular point groups are not sufficient for this 
task: They are tailored to "local" molecular properties which are located at, or close to stationary points on the potential energy surface, e.g. they provide the symmetry properties of normal modes, i.e. vibrations with small amplitudes about the minimum structures [95]. In contrast, the present class of molecules allows for large amplitude motions after photo-excitation, i.e. torsions which cover domains on the potential energy surface even far away from the "local" minimum structures. As it was shown by Longuet-Higgins [96] on the basis of preliminary works of Hougen [97,98] it is, however, possible to define a group which can be used for the classification of the molecular states for molecules allowing for internal large amplitude motions: the molecular symmetry (MS) group [99]. It contains all the so-called "feasible" permutations $(P)$ and permutation-inversions $(P)^{*}$ of identical nuclei. From an experimental point of view, the term "feasible" means, qualitatively speaking, that the permutations and permutation-inversions are observable on the time scale which is associated with the resolution of the experiment. From the perspective of a quantum dynamicist, the permutations and permutation-inversions are feasible if they can be simulated by time-dependent wavepackets, within the time scales of the process which is investigated. For the present class of molecules, with CCD as a model representative, those wavepackets are torsional wavepackets. We shall show, as one of the important discoveries of this paper, that the MS group for this class of molecules is $\mathrm{G}_{16}^{\mathrm{A}}$. To the best of our knowledge, this Abelian MS group has not been applied previously to any other molecule. In any case, it is different from the non-Abelian group $\mathrm{G}_{16}$ which has been employed in Refs. [100,101] for the description of the molecular symmetry of ethylene-like molecules with just one feasible torsion. In order to distinguish the two MS-groups, we shall call the present Abelian group $\mathrm{G}_{16}^{\mathrm{A}}$, with superscript " $\mathrm{A}$ " representing "Abelian". Since $\mathrm{G}_{16}^{\mathrm{A}}$ is a new group, we also will report various important properties, e.g. the irreducible presentations (IREPs), to facilitate the task of finding the NSIs of the model CCD, as representative of the present class of molecules.

The rest of this paper is organized as follows: In Sect. 2 the theory, the model and the techniques are described necessary to identify the NSIs for the present class of molecules with the properties (i) and (ii), using CCD as an example. In Sect. 3 the results are presented and discussed. The conclusions and an outlook to photo-chemical and quantum dynamical applications and extensions are contained in Sect. 4. This includes a discussion of the implications the results of this paper have for further investigations on photo-excited torsions.

\section{Model and techniques}

This section is divided into four subsections. We begin with specifying the Hamiltonian in full dimensionality and derive the corresponding molecular symmetry group $G_{16}^{A}$. In Sect. 2.2 we proceed with the reduction of the Hamiltonian from full dimensionality to the $2 \mathrm{~d}$ model for the two torsional dof in the frame of the Born-Oppenheimer approximation. Sect. 2.3 presents details for the quantum chemical calculations of the "first" molecular equilibrium structure and the neighboring domains of the potential energy surfaces in the electronic ground and excited states, and for the evaluations of the torsional wavefunctions. The section closes with Sect. 2.4 which discusses how the NSIs 
of an arbitrary molecule can be identified with the help of the MS group and the symmetrization postulate. In every Subsection, CCD serves as an example for the present class of molecules, see items (i), (ii) in Sect. 1, with the notations which are explained in the legend of Fig. 1. We use SI units throughout this section.

\subsection{Hamiltonian in full dimensionality, and derivation of the molecular symmetry group}

The present class of molecules, e.g. CCD, suggests divisions into fragments labeled (0)-(6), as shown in Fig. 1. It is convenient to write the complete Hamiltonian with notation that corresponds to this division,

$$
\hat{H}=\hat{H}^{\{1\}}+\hat{H}^{\{2\}}+\hat{T}_{\mathrm{e}}+\hat{V}_{\mathrm{ee}}
$$

where

$$
\hat{H}^{[1\}}=\sum_{i=0}^{6} \hat{H}_{i}^{\{1\}}
$$

and

$$
\hat{H}^{\{2\}}=\sum_{i=0}^{6} \sum_{j=i}^{6} \hat{H}_{i j}^{\{2\}}
$$

denote contributions which can be attributed to the individual (superscript $\{1\}$ ) nuclei in fragments $i=0, \ldots, 6$, or to the interactions of two (superscript $\{2\}$ ) nuclei, one of them in fragment $i=0, \ldots, 6$ and the other one in fragment $j=i, \ldots, 6$. The numbers of nuclei in fragments $i$ are denoted with $N_{i}$, so that the total number of nuclei is $N_{n}=\sum_{i=0}^{6} N_{i}$. The terms $\hat{T}_{\mathrm{e}}$ and $\hat{V}_{\text {ee }}$ account for the kinetic energies and for the Coulomb interactions of the total number of $N_{\mathrm{e}}$ electrons - these are delocalized, so they are, of course, not attributed to any molecular fragments,

$$
\begin{aligned}
& \hat{T}_{\mathrm{e}}=\sum_{n=1}^{N_{\mathrm{e}}}-\frac{\hbar^{2}}{2 m_{\mathrm{e}}} \nabla_{n}^{2} \\
& \hat{V}_{\mathrm{ee}}=\sum_{m=1}^{N_{\mathrm{e}}-1} \sum_{n=m+1}^{N_{\mathrm{e}}} \frac{e_{0}^{2}}{4 \pi \epsilon_{0}\left|\vec{r}_{m}-\vec{r}_{n}\right|}
\end{aligned}
$$

where $\vec{r}_{m}$ denotes the position of electron $m, m=1, \ldots, N_{\mathrm{e}}$. Likewise,

$$
\hat{H}_{i j}^{\{2\}}=\sum_{k=1}^{N_{i}} \sum_{l=1}^{N_{j}} \frac{Z_{i k} Z_{j l} e_{0}^{2}}{4 \pi \epsilon_{0}\left|\vec{R}_{i k}-\vec{R}_{j l}\right|}
$$

are the Coulomb interactions between all the nuclei (labeled with $k$ ) of fragment $(i)$ at $\vec{R}_{i k}$ with all the nuclei (labeled with $l$ ) of fragment $(j)$ at $\vec{R}_{j l}$. For identical fragments $(i=j)$, the double sum in Eq. (6) runs from $k=1$ to $N_{i}-1$ and from $l=k+1$ to $N_{i}$. 
For simplicity, we neglect spin orbit interactions or any other interactions involving electronic or nuclear spins. Correspondingly,

$$
\begin{aligned}
\hat{H}_{i}^{\{1\}} & =\hat{T}_{i}+\hat{V}_{i, \mathrm{e}} \\
& =\sum_{k=1}^{N_{i}}-\frac{\hbar^{2}}{2 M_{i k}} \nabla_{m}^{2}-\sum_{k=1}^{N_{i}} \sum_{m=1}^{N_{\mathrm{e}}} \frac{Z_{i k} e_{0}^{2}}{4 \pi \epsilon_{0}\left|\vec{R}_{i k}-\vec{r}_{m}\right|}
\end{aligned}
$$

is the sum of the kinetic energies of all the nuclei in fragment $(i)$ plus their Coulomb interactions with all electrons. Here $M_{i k}, Z_{i k} e_{0}$ and $\vec{R}_{i k}$ denote the mass, the charge and the position of nucleus $k$ in fragment $(i)$. Note the consistent use of Capital and small letters for nuclear and electronic properties, respectively; accordingly, nuclear and electronic wavefunctions will be written as $\Psi$ and $\psi$, respectively, angles of intramolecular rotations or torsions are denoted $\Phi$, etc.

In order to determine the MS group of CCD or any other representative of the class of molecules which satisfy the criteria (i), (ii) from Sect. 1, we consider the scenario where, on one hand, the total energy $E$ of the molecule is sufficiently low so that the bonds in fragments $(0)-(6)$ cannot break, but on the other hand, $E$ is sufficiently high so that the equivalent fragments (1) and (2) or/and (3) and (4) or/and (5) and (6) can be exchanged at once. In other words: the nuclei in fragment $(i)$ keep their labels $k=1-N_{i}$, without any exchange of the nuclei within the fragments, but all the nuclei $k=1, \ldots, N_{i}$ in fragment $(i)=(1),(3)$ or (5) may be exchanged by the equivalent nuclei $k=1, \ldots, N_{j}$ in fragments $(j)=(2)$, (4) or (6), respectively, e.g. by intramolecular rotations of the segments (A), (B), or (C). The corresponding permutations of the fragments will be denoted with (12), (34) and (56), respectively. In addition, simultaneous exchanges of two fragments, either (1) and (2) and (3) and (4), or (1) and (2) and (5) and (6), or (3) and (4) and (5) and (6), are denoted (12)(34), (12)(56) and (34)(56), respectively. The exchange of all fragments at once is denoted (12)(34)(56). Obviously, the Hamiltonian Eq. (1) is robust with respect to these permutations of fragments, or in other words, the set $\mathfrak{P}$ of permutations $(P)$

$$
\mathfrak{P}=\{E,(12),(34),(56),(12)(34),(12)(56),(34)(56),(12)(34)(56)\},
$$

with $E$ denoting the identity, commutes with the Hamiltonian Eq. (1),

$$
[\hat{H}, \mathcal{R}]=0 \quad \text { for } \mathcal{R} \in \mathfrak{P} .
$$

Moreover, the Hamiltonian from Eq. (1) is invariant with respect to inversion $E^{*}$ of all electronic and nuclear coordinates,

$$
\left[\hat{H}, E^{*}\right]=0 \text {. }
$$

As a consequence, $\hat{H}$ commutes with all elements $\mathcal{R} \in \mathfrak{P}$ and with eight feasible permutation-inversions, which can be written as $(P) E^{*} \equiv(P)^{*}$. Since it is impossible to find any feasible permutation or permutation-inversion $(P)^{*}$ with $(P) \notin \mathfrak{P}$ without 
Table 1. The multiplication table for the group $\mathrm{G}_{16}^{\mathrm{A}}$. The abbreviations are: $F=(12) ; G=(34) ; H=$ (12)(34); $I=(56) ; \quad J=(12)(56) ; K=(34)(56) ; L=(12)(34)(56) ; F^{*}=(12)^{*} ; G^{*}=(34)^{*} ; H^{*}=$ $(12)(34)^{*} ; I^{*}=(56)^{*} ; J^{*}=(12)(56)^{*} ; K^{*}=(34)(56)^{*} ; L^{*}=(12)(34)(56)^{*}$.

\begin{tabular}{|c|c|c|c|c|c|c|c|c|c|c|c|c|c|c|c|c|}
\hline $\mathrm{G}_{16}^{A}$ & $E$ & $F$ & $G$ & $H$ & $I$ & $J$ & $K$ & $L$ & $E^{*}$ & $F^{*}$ & $G^{*}$ & $H^{*}$ & $I^{*}$ & $J^{*}$ & $K^{*}$ & $L^{*}$ \\
\hline$E$ & $E$ & $F$ & $G$ & $H$ & $I$ & $J$ & $K$ & $L$ & $E^{*}$ & $F^{*}$ & $G^{*}$ & $H^{*}$ & $I^{*}$ & $J^{*}$ & $K^{*}$ & $L^{*}$ \\
\hline$F$ & $F$ & $E$ & $H$ & $G$ & $J$ & $I$ & $L$ & $K$ & $F^{*}$ & $E^{*}$ & $H^{*}$ & $G^{*}$ & $J^{*}$ & $I^{*}$ & $L^{*}$ & $K^{*}$ \\
\hline$G$ & $G$ & $H$ & $E$ & $F$ & $K$ & $L$ & $I$ & $J$ & $G^{*}$ & $H^{*}$ & $E^{*}$ & $F^{*}$ & $K^{*}$ & $L^{*}$ & $I^{*}$ & $J^{*}$ \\
\hline$H$ & $H$ & $G$ & $F$ & $E$ & $L$ & $K$ & $J$ & $I$ & $H^{*}$ & $G^{*}$ & $F^{*}$ & $E^{*}$ & $L^{*}$ & $K^{*}$ & $J^{*}$ & $I^{*}$ \\
\hline$I$ & $I$ & $J$ & $K$ & $L$ & $E$ & $F$ & $G$ & $H$ & $I^{*}$ & $J^{*}$ & $K^{*}$ & $L^{*}$ & $E^{*}$ & $F^{*}$ & $G^{*}$ & $H^{*}$ \\
\hline$J$ & $J$ & $I$ & $L$ & $K$ & $F$ & $E$ & $H$ & $G$ & $J^{*}$ & $I^{*}$ & $L^{*}$ & $K^{*}$ & $F^{*}$ & $E^{*}$ & $H^{*}$ & $G^{*}$ \\
\hline$K$ & $K$ & $L$ & $I$ & $J$ & $G$ & $H$ & $E$ & $F$ & $K^{*}$ & $L^{*}$ & $I^{*}$ & $J^{*}$ & $G^{*}$ & $H^{*}$ & $E^{*}$ & $F^{*}$ \\
\hline$L$ & $L$ & $K$ & $J$ & $I$ & $H$ & $G$ & $F$ & $E$ & $L^{*}$ & $K^{*}$ & $J^{*}$ & $I^{*}$ & $H^{*}$ & $G^{*}$ & $F^{*}$ & $E^{*}$ \\
\hline$E^{*}$ & $E^{*}$ & $F^{*}$ & $G^{*}$ & $H^{*}$ & $I^{*}$ & $J^{*}$ & $K^{*}$ & $L^{*}$ & $E$ & $F$ & $G$ & $H$ & $I$ & $J$ & $K$ & $L$ \\
\hline$F^{*}$ & $F^{*}$ & $E^{*}$ & $H^{*}$ & $G^{*}$ & $J^{*}$ & $I^{*}$ & $L^{*}$ & $K^{*}$ & $F$ & $E$ & $H$ & $G$ & $J$ & $I$ & $L$ & $K$ \\
\hline$G^{*}$ & $G^{*}$ & $H^{*}$ & $E^{*}$ & $F^{*}$ & $K^{*}$ & $L^{*}$ & $I^{*}$ & $J^{*}$ & $G$ & $H$ & $E$ & $F$ & $K$ & $L$ & $I$ & $J$ \\
\hline$H^{*}$ & $H^{*}$ & $G^{*}$ & $F^{*}$ & $E^{*}$ & $L^{*}$ & $K^{*}$ & $J^{*}$ & $I^{*}$ & $H$ & $G$ & $F$ & $E$ & $L$ & $K$ & $J$ & $I$ \\
\hline$I^{*}$ & $I^{*}$ & $J^{*}$ & $K^{*}$ & $L^{*}$ & $E^{*}$ & $F^{*}$ & $G^{*}$ & $H^{*}$ & $I$ & $J$ & $K$ & $L$ & $E$ & $F$ & $G$ & $H$ \\
\hline$J^{*}$ & $J^{*}$ & $I^{*}$ & $L^{*}$ & $K^{*}$ & $F^{*}$ & $E^{*}$ & $H^{*}$ & $G^{*}$ & $J$ & $I$ & $L$ & $K$ & $F$ & $E$ & $H$ & $G$ \\
\hline$K^{*}$ & $K^{*}$ & $L^{*}$ & $I^{*}$ & $J^{*}$ & $G^{*}$ & $H^{*}$ & $E^{*}$ & $F^{*}$ & $K$ & $L$ & $I$ & $J$ & $G$ & $H$ & $E$ & $F$ \\
\hline$L^{*}$ & $L^{*}$ & $K^{*}$ & $J^{*}$ & $I^{*}$ & $H^{*}$ & $G^{*}$ & $F^{*}$ & $E^{*}$ & $L$ & $K$ & $J$ & $I$ & $H$ & $G$ & $F$ & $E$ \\
\hline
\end{tabular}

breaking bonds within one fragment, we can write the set of sixteen feasible permutations and permutation-inversions which commute with $\hat{H}$, Eq. (1), as

$$
\begin{aligned}
\mathrm{G}_{16}^{\mathrm{A}} & =\mathfrak{P} \otimes\left\{E, E^{*}\right\} \\
& =\left\{E,(12), \ldots,(12)(34)(56)^{*}\right\}
\end{aligned}
$$

It can be verified easily that the set Eq. (11) forms a group - the MS group of CCD, $\mathrm{G}_{16}^{\mathrm{A}}$. Its multiplication table is given in Table 1. Note, that $\mathrm{G}_{16}^{\mathrm{A}}$ is the MS group not only of CCD, but also for all other molecules which satisfy the criteria (i) and (ii), $c f$. Sect. 1.

As is well known from group theory $[95,99,102]$, groups are uniquely defined by their presentations, i.e. the generators of the groups and the set of relations between them. For $\mathrm{G}_{16}^{\mathrm{A}}$

$$
\mathrm{G}_{16}^{\mathrm{A}}=\left\langle(12),(34),(56), E^{*} \mid(12)^{2}=(34)^{2}=(56)^{2}=\left(E^{*}\right)^{2}=E\right\rangle,
$$

where $(12)^{2}$ means (12)(12), etc. Thus, $\mathrm{G}_{16}^{\mathrm{A}}$ is generated by the four elements (12), (34), (56), $E^{*}$. With the help of Eq. (13) alone, we can generate the complete group $\mathrm{G}_{16}^{\mathrm{A}}$, which will be very helpful in Sect. 2.4 , as we identify the symmetry adapted torsional eigenfunctions. Additionally, since $\mathrm{G}_{16}^{\mathrm{A}}$ can be written as

$$
\mathrm{G}_{16}^{\mathrm{A}}=\{E,(12)\} \otimes\{E,(34)\} \otimes\{E,(56)\} \otimes\left\{E, E^{*}\right\},
$$

we can directly deduce from the fundamental theorem of finite Abelian groups that $\mathrm{G}_{16}^{\mathrm{A}}$ must be Abelian [102].

The character table of $\mathrm{G}_{16}^{\mathrm{A}}$, displaying all its irreducible representations (IREPs), is shown in Table 2. The multiplication table, containing all cogitable direct products 
Table 2. The character table of the group $\mathrm{G}_{16}^{\mathrm{A}}$. The abbreviations are: $F=(12) ; G=(34) ; H=(12)(34)$; $I=(56) ; J=(12)(56) ; K=(34)(56) ; L=(12)(34)(56) ; F^{*}=(12)^{*} ; G^{*}=(34)^{*} ; H^{*}=(12)(34)^{*} ; I^{*}=$ $(56)^{*} ; J^{*}=(12)(56)^{*} ; K^{*}=(34)(56)^{*} ; L^{*}=(12)(34)(56)^{*}$.

\begin{tabular}{lrrrrrrrrrrrrrrrr}
\hline $\mathrm{G}_{16}^{\mathrm{A}}$ & $E$ & $F$ & $G$ & $H$ & $I$ & $J$ & $K$ & $L$ & $E^{*}$ & $F^{*}$ & $G^{*}$ & $H^{*}$ & $I^{*}$ & $J^{*}$ & $K^{*}$ & $L^{*}$ \\
\hline$\Gamma_{1}^{+}$ & 1 & 1 & 1 & 1 & 1 & 1 & 1 & 1 & 1 & 1 & 1 & 1 & 1 & 1 & 1 & 1 \\
$\Gamma_{2}^{+}$ & 1 & 1 & 1 & 1 & -1 & -1 & -1 & -1 & 1 & 1 & 1 & 1 & -1 & -1 & -1 & -1 \\
$\Gamma_{3}^{+}$ & 1 & 1 & -1 & -1 & 1 & 1 & -1 & -1 & 1 & 1 & -1 & -1 & 1 & 1 & -1 & -1 \\
$\Gamma_{4}^{+}$ & 1 & 1 & -1 & -1 & -1 & -1 & 1 & 1 & 1 & 1 & -1 & -1 & -1 & -1 & 1 & 1 \\
$\Gamma_{5}^{+}$ & 1 & -1 & 1 & -1 & 1 & -1 & 1 & -1 & 1 & -1 & 1 & -1 & 1 & -1 & 1 & -1 \\
$\Gamma_{6}^{+}$ & 1 & -1 & 1 & -1 & -1 & 1 & -1 & 1 & 1 & -1 & 1 & -1 & -1 & 1 & -1 & 1 \\
$\Gamma_{7}^{+}$ & 1 & -1 & -1 & 1 & 1 & -1 & -1 & 1 & 1 & -1 & -1 & 1 & 1 & -1 & -1 & 1 \\
$\Gamma_{8}^{+}$ & 1 & -1 & -1 & 1 & -1 & 1 & 1 & -1 & 1 & -1 & -1 & 1 & -1 & 1 & 1 & -1 \\
$\Gamma_{1}^{-}$ & 1 & 1 & 1 & 1 & 1 & 1 & 1 & 1 & -1 & -1 & -1 & -1 & -1 & -1 & -1 & -1 \\
$\Gamma_{2}^{-}$ & 1 & 1 & 1 & 1 & -1 & -1 & -1 & -1 & -1 & -1 & -1 & -1 & 1 & 1 & 1 & 1 \\
$\Gamma_{3}^{-}$ & 1 & 1 & -1 & -1 & 1 & 1 & -1 & -1 & -1 & -1 & 1 & 1 & -1 & -1 & 1 & 1 \\
$\Gamma_{4}^{-}$ & 1 & 1 & -1 & -1 & -1 & -1 & 1 & 1 & -1 & -1 & 1 & 1 & 1 & 1 & -1 & -1 \\
$\Gamma_{5}^{-}$ & 1 & -1 & 1 & -1 & 1 & -1 & 1 & -1 & -1 & 1 & -1 & 1 & -1 & 1 & -1 & 1 \\
$\Gamma_{6}^{-}$ & 1 & -1 & 1 & -1 & -1 & 1 & -1 & 1 & -1 & 1 & -1 & 1 & 1 & -1 & 1 & -1 \\
$\Gamma_{7}^{-}$ & 1 & -1 & -1 & 1 & 1 & -1 & -1 & 1 & -1 & 1 & 1 & -1 & -1 & 1 & 1 & -1 \\
$\Gamma_{8}^{-}$ & 1 & -1 & -1 & 1 & -1 & 1 & 1 & -1 & -1 & 1 & 1 & -1 & 1 & -1 & -1 & 1 \\
\hline
\end{tabular}

of the IREPs of $\mathrm{G}_{16}^{\mathrm{A}}$ is shown in Table 3. From the characters $\chi^{\Gamma_{i}}[\mathcal{R}]$ for operation $\mathcal{R}$ of IREP $\Gamma_{i}$ in Table 2, we obtain molecular symmetry projection operators for the irreducible representations

$$
\hat{\mathcal{P}}^{\Gamma_{i}}=\frac{1}{16} \sum_{\mathcal{R}} \chi^{\Gamma_{i}}[\mathcal{R}] \mathcal{R} \quad \text { with } \quad \mathcal{R} \in \mathrm{G}_{16}^{\mathrm{A}} .
$$

The fact that the Hamiltonian Eq. (1) commutes with all operations $\mathcal{R}$ of the group $\mathrm{G}_{16}^{\mathrm{A}}$ implies that the projection operators $\hat{\mathcal{P}}^{\Gamma_{i}}$ also commute with $\hat{H}$,

$$
\left[\hat{H}, \hat{\mathcal{P}}^{\Gamma_{i}}\right]=0 \text {. }
$$

Moreover, by construction Eq. (15), all the projection operators $\hat{\mathcal{P}}^{\Gamma_{i}}$ are mutually orthogonal, i.e. they commute with each other $[99,102]$,

$$
\left[\hat{\mathcal{P}}^{\Gamma_{i}}, \hat{\mathcal{P}}^{\Gamma_{j}}\right]=0 \text {. }
$$

As a consequence, all the molecular eigenstates are labeled simultaneously by the eigenvalues of $\hat{H}$ and all the $\hat{\mathcal{P}}^{\Gamma_{i}}$. It follows that all the molecular eigenstates of CCD are specified by their energy (the eigenvalue of $\hat{H}$ ) and by one of the sixteen IREPs which are listed in the character table. This simplified notation - IREP label instead of sixteen eigenvalues of the individual $\hat{\mathcal{P}}^{\Gamma_{i}}, i=1, \ldots, 16-$ is a consequence of the orthogonality expressed in Eq. (17), and the idempotence of the $\hat{\mathcal{P}}^{\Gamma_{i}}$

$$
\hat{\mathcal{P}}^{\Gamma_{i}} \hat{\mathcal{P}}^{\Gamma_{i}}=\hat{\mathcal{P}}^{\Gamma_{i}}
$$


Table 3. Product table for the group $\mathrm{G}_{16}^{\mathrm{A}}$.

\begin{tabular}{|c|c|c|c|c|c|c|c|c|c|c|c|c|c|c|c|c|}
\hline $\mathrm{G}_{16}^{\mathrm{A}}$ & $\Gamma_{1}^{+}$ & $\Gamma_{2}^{+}$ & $\Gamma_{3}^{+}$ & $\Gamma_{4}^{+}$ & $\Gamma_{5}^{+}$ & $\Gamma_{6}^{+}$ & $\Gamma_{7}^{+}$ & $\Gamma_{8}^{+}$ & $\Gamma_{1}^{-}$ & $\Gamma_{2}^{-}$ & $\Gamma_{3}^{-}$ & $\Gamma_{4}^{-}$ & $\Gamma_{5}^{-}$ & $\Gamma_{6}^{-}$ & $\Gamma_{7}^{-}$ & $\Gamma_{8}^{-}$ \\
\hline$\Gamma_{1}^{+}$ & $\Gamma_{1}^{+}$ & $\Gamma_{2}^{+}$ & $\Gamma_{3}^{+}$ & $\Gamma_{4}^{+}$ & $\Gamma_{5}^{+}$ & $\Gamma_{6}^{+}$ & $\Gamma_{7}^{+}$ & $\Gamma_{8}^{+}$ & $\Gamma_{1}^{-}$ & $\Gamma_{2}^{-}$ & $\Gamma_{3}^{-}$ & $\Gamma_{4}^{-}$ & $\Gamma_{5}^{-}$ & $\Gamma_{6}^{-}$ & $\Gamma_{7}^{-}$ & $\Gamma_{8}^{-}$ \\
\hline$\Gamma_{2}^{+}$ & $\Gamma_{2}^{+}$ & $\Gamma_{1}^{+}$ & $\Gamma_{4}^{+}$ & $\Gamma_{3}^{+}$ & $\Gamma_{6}^{+}$ & $\Gamma_{5}^{+}$ & $\Gamma_{8}^{+}$ & $\Gamma_{7}^{+}$ & $\Gamma_{2}^{-}$ & $\Gamma_{1}^{-}$ & $\Gamma_{4}^{-}$ & $\Gamma_{3}^{-}$ & $\Gamma_{6}^{-}$ & $\Gamma_{5}^{-}$ & $\Gamma_{8}^{-}$ & $\Gamma_{7}^{-}$ \\
\hline$\Gamma_{3}^{+}$ & $\Gamma_{3}^{+}$ & $\Gamma_{4}^{+}$ & $\Gamma_{1}^{+}$ & $\Gamma_{2}^{+}$ & $\Gamma_{7}^{+}$ & $\Gamma_{8}^{+}$ & $\Gamma_{5}^{+}$ & $\Gamma_{6}^{+}$ & $\Gamma_{3}^{-}$ & $\Gamma_{4}^{-}$ & $\Gamma_{1}^{-}$ & $\Gamma_{2}^{-}$ & $\Gamma_{7}^{-}$ & $\Gamma_{8}^{-}$ & $\Gamma_{5}^{-}$ & $\Gamma_{6}^{-}$ \\
\hline$\Gamma_{4}^{+}$ & $\Gamma_{4}^{+}$ & $\Gamma_{3}^{+}$ & $\Gamma_{2}^{+}$ & $\Gamma_{1}^{+}$ & $\Gamma_{8}^{+}$ & $\Gamma_{7}^{+}$ & $\Gamma_{6}^{+}$ & $\Gamma_{5}^{+}$ & $\Gamma_{4}^{-}$ & $\Gamma_{3}^{-}$ & $\Gamma_{2}^{-}$ & $\Gamma_{1}^{-}$ & $\Gamma_{8}^{-}$ & $\Gamma_{7}^{-}$ & $\Gamma_{6}^{-}$ & $\Gamma_{5}^{-}$ \\
\hline$\Gamma_{5}^{+}$ & $\Gamma_{5}^{+}$ & $\Gamma_{6}^{+}$ & $\Gamma_{7}^{+}$ & $\Gamma_{8}^{+}$ & $\Gamma_{1}^{+}$ & $\Gamma_{2}^{+}$ & $\Gamma_{3}^{+}$ & $\Gamma_{4}^{+}$ & $\Gamma_{5}^{-}$ & $\Gamma_{6}^{-}$ & $\Gamma_{7}^{-}$ & $\Gamma_{8}^{-}$ & $\Gamma_{1}^{-}$ & $\Gamma_{2}^{-}$ & $\Gamma_{3}^{-}$ & $\Gamma_{4}^{-}$ \\
\hline$\Gamma_{6}^{+}$ & $\Gamma_{6}^{+}$ & $\Gamma_{5}^{+}$ & $\Gamma_{8}^{+}$ & $\Gamma_{7}^{+}$ & $\Gamma_{2}^{+}$ & $\Gamma_{1}^{+}$ & $\Gamma_{4}^{+}$ & $\Gamma_{3}^{+}$ & $\Gamma_{6}^{-}$ & $\Gamma_{5}^{-}$ & $\Gamma_{8}^{-}$ & $\Gamma_{7}^{-}$ & $\Gamma_{2}^{-}$ & $\Gamma_{1}^{-}$ & $\Gamma_{4}^{-}$ & $\Gamma_{3}^{-}$ \\
\hline$\Gamma_{7}^{+}$ & $\Gamma_{7}^{+}$ & $\Gamma_{8}^{+}$ & $\Gamma_{5}^{+}$ & $\Gamma_{6}^{+}$ & $\Gamma_{3}^{+}$ & $\Gamma_{4}^{+}$ & $\Gamma_{1}^{+}$ & $\Gamma_{2}^{+}$ & $\Gamma_{7}^{-}$ & $\Gamma_{8}^{-}$ & $\Gamma_{5}^{-}$ & $\Gamma_{6}^{-}$ & $\Gamma_{3}^{-}$ & $\Gamma_{4}^{-}$ & $\Gamma_{1}^{-}$ & $\Gamma_{2}^{-}$ \\
\hline$\Gamma_{8}^{+}$ & $\Gamma_{8}^{+}$ & $\Gamma_{7}^{+}$ & $\Gamma_{6}^{+}$ & $\Gamma_{5}^{+}$ & $\Gamma_{4}^{+}$ & $\Gamma_{3}^{+}$ & $\Gamma_{2}^{+}$ & $\Gamma_{1}^{+}$ & $\Gamma_{8}^{-}$ & $\Gamma_{7}^{-}$ & $\Gamma_{6}^{-}$ & $\Gamma_{5}^{-}$ & $\Gamma_{4}^{-}$ & $\Gamma_{3}^{-}$ & $\Gamma_{2}^{-}$ & $\Gamma_{1}^{-}$ \\
\hline$\Gamma_{1}^{-}$ & $\Gamma_{1}^{-}$ & $\Gamma_{2}^{-}$ & $\Gamma_{3}^{-}$ & $\Gamma_{4}^{-}$ & $\Gamma_{5}^{-}$ & $\Gamma_{6}^{-}$ & $\Gamma_{7}^{-}$ & $\Gamma_{8}^{-}$ & $\Gamma_{1}^{+}$ & $\Gamma_{2}^{+}$ & $\Gamma_{3}^{+}$ & $\Gamma_{4}^{+}$ & $\Gamma_{5}^{+}$ & $\Gamma_{6}^{+}$ & $\Gamma_{7}^{+}$ & $\Gamma_{8}^{+}$ \\
\hline$\Gamma_{2}^{-}$ & $\Gamma_{2}^{-}$ & $\Gamma_{1}^{-}$ & $\Gamma_{4}^{-}$ & $\Gamma_{3}^{-}$ & $\Gamma_{6}^{-}$ & $\Gamma_{5}^{-}$ & $\Gamma_{8}^{-}$ & $\Gamma_{7}^{-}$ & $\Gamma_{2}^{+}$ & $\Gamma_{1}^{+}$ & $\Gamma_{4}^{+}$ & $\Gamma_{3}^{+}$ & $\Gamma_{6}^{+}$ & $\Gamma_{5}^{+}$ & $\Gamma_{8}^{+}$ & $\Gamma_{7}^{+}$ \\
\hline$\Gamma_{3}^{-}$ & $\Gamma_{3}^{-}$ & $\Gamma_{4}^{-}$ & $\Gamma_{1}^{-}$ & $\Gamma_{2}^{-}$ & $\Gamma_{7}^{-}$ & $\Gamma_{8}^{-}$ & $\Gamma_{5}^{-}$ & $\Gamma_{6}^{-}$ & $\Gamma_{3}^{+}$ & $\Gamma_{4}^{+}$ & $\Gamma_{1}^{+}$ & $\Gamma_{2}^{+}$ & $\Gamma_{7}^{+}$ & $\Gamma_{8}^{+}$ & $\Gamma_{5}^{+}$ & $\Gamma_{6}^{+}$ \\
\hline$\Gamma_{4}^{-}$ & $\Gamma_{4}^{-}$ & $\Gamma_{3}^{-}$ & $\Gamma_{2}^{-}$ & $\Gamma_{1}^{-}$ & $\Gamma_{8}^{-}$ & $\Gamma_{7}^{-}$ & $\Gamma_{6}^{-}$ & $\Gamma_{5}^{-}$ & $\Gamma_{4}^{+}$ & $\Gamma_{3}^{+}$ & $\Gamma_{2}^{+}$ & $\Gamma_{1}^{+}$ & $\Gamma_{8}^{+}$ & $\Gamma_{7}^{+}$ & $\Gamma_{6}^{+}$ & $\Gamma_{5}^{+}$ \\
\hline$\Gamma_{5}^{-}$ & $\Gamma_{5}^{-}$ & $\Gamma_{6}^{-}$ & $\Gamma_{7}^{-}$ & $\Gamma_{8}^{-}$ & $\Gamma_{1}^{-}$ & $\Gamma_{2}^{-}$ & $\Gamma_{3}^{-}$ & $\Gamma_{4}^{-}$ & $\Gamma_{5}^{+}$ & $\Gamma_{6}^{+}$ & $\Gamma_{7}^{+}$ & $\Gamma_{8}^{+}$ & $\Gamma_{1}^{+}$ & $\Gamma_{2}^{+}$ & $\Gamma_{3}^{+}$ & $\Gamma_{4}^{+}$ \\
\hline$\Gamma_{6}^{-}$ & $\Gamma_{6}^{-}$ & $\Gamma_{5}^{-}$ & $\Gamma_{8}^{-}$ & $\Gamma_{7}^{-}$ & $\Gamma_{2}^{-}$ & $\Gamma_{1}^{-}$ & $\Gamma_{4}^{-}$ & $\Gamma_{3}^{-}$ & $\Gamma_{6}^{+}$ & $\Gamma_{5}^{+}$ & $\Gamma_{8}^{+}$ & $\Gamma_{7}^{+}$ & $\Gamma_{2}^{+}$ & $\Gamma_{1}^{+}$ & $\Gamma_{4}^{+}$ & $\Gamma_{3}^{+}$ \\
\hline$\Gamma_{7}^{-}$ & $\Gamma_{7}^{-}$ & $\Gamma_{8}^{-}$ & $\Gamma_{5}^{-}$ & $\Gamma_{6}^{-}$ & $\Gamma_{3}^{-}$ & $\Gamma_{4}^{-}$ & $\Gamma_{1}^{-}$ & $\Gamma_{2}^{-}$ & $\Gamma_{7}^{+}$ & $\Gamma_{8}^{+}$ & $\Gamma_{5}^{+}$ & $\Gamma_{6}^{+}$ & $\Gamma_{3}^{+}$ & $\Gamma_{4}^{+}$ & $\Gamma_{1}^{+}$ & $\Gamma_{2}^{+}$ \\
\hline$\Gamma_{8}^{-}$ & $\Gamma_{8}^{-}$ & $\Gamma_{7}^{-}$ & $\Gamma_{6}^{-}$ & $\Gamma_{5}^{-}$ & $\Gamma_{4}^{-}$ & $\Gamma_{3}^{-}$ & $\Gamma_{2}^{-}$ & $\Gamma_{1}^{-}$ & $\Gamma_{8}^{+}$ & $\Gamma_{7}^{+}$ & $\Gamma_{6}^{+}$ & $\Gamma_{5}^{+}$ & $\Gamma_{4}^{+}$ & $\Gamma_{3}^{+}$ & $\Gamma_{2}^{+}$ & $\Gamma_{1}^{+}$ \\
\hline
\end{tabular}

which is imposed by construction Eq. (15) [99,102]. Relation Eq. (18) implies that the eigenvalues of $\hat{\mathcal{P}}^{\Gamma_{i}}$ are either 1 or 0 . Furthermore, the orthogonality Eq. (17) suggests that if the eigenvalue of $\hat{\mathcal{P}}^{\Gamma_{i}}$ is equal to 1 , then it is equal to zero for all other projection operators $\hat{\mathcal{P}}^{\Gamma_{j}}, j \neq i$. The set of projection operators $\hat{\mathcal{P}}^{\Gamma_{i}}$ is therefore associated with symmetry quantum numbers $\{0,0, \ldots, 0,1,0, \ldots, 0\}$, where the position $i$ of the single number " 1 " is mapped in a unique, bijective manner to the IREP of the projection operator $\hat{\mathcal{P}}^{\Gamma_{i}}$. The specification of the IREP is thus equivalent to the list of all sixteen quantum numbers of the projection operators.

For the identification of the NSIs of CCD, however, only the permutation subgroup $\mathrm{G}^{\mathrm{PSMS}}\left[\mathrm{G}_{16}^{\mathrm{A}}\right]$ is relevant [49]. As can be seen by comparison of Eqs. (82 and (11) this group is identical to the set $\mathfrak{P}$, with only eight instead of sixteen elements. The character table of this group is shown in Table 4. This character table, Table 4, already provides the answer to one of the questions which have been raised in the Introduction, i.e. CCD has at most eight different NSIs which are characterized by the irreducible representations IREPs of their nuclear spin wavefunctions. Moreover, since $\mathrm{G}^{\mathrm{PSMS}}\left[\mathrm{G}_{16}^{\mathrm{A}}\right]$ is Abelian (because it is a subgroup of the Abelian group $\mathrm{G}_{16}^{\mathrm{A}}$ ), we can utilize its character table for direct construction of the symmetry-adapted torsional and nuclear spin wavefunctions with the help of projection operators $\hat{\mathcal{P}}^{\Gamma_{i}}$ for the irreducible representation $\Gamma_{i}$. The definition of these symmetry projection operators,

$$
\hat{\mathcal{P}}^{\Gamma_{i}}=\frac{1}{8} \sum_{\mathcal{R}} \chi^{\Gamma_{i}}[\mathcal{R}] \mathcal{R} \quad \text { with } \quad \mathcal{R} \in \mathrm{G}^{\mathrm{PSMS}}\left[\mathrm{G}_{16}^{\mathrm{A}}\right]
$$

and all their properties (see Eqs. 16-18) are entirely analogous to the projection operators of $\mathrm{G}_{16}^{\mathrm{A}}$. We shall employ these projection operators below, in order to generate symmetry adapted torsional wavefunctions. In practice, we shall start by calculation of a wavefunction $\Psi_{l}$, which is localized (subscript "l") in a single potential well so that 
Table 4. The character table of $\mathrm{G}^{\mathrm{PSMS}}\left[\mathrm{G}_{16}^{\mathrm{A}}\right]$, the permutation subgroup of the MS group of $\mathrm{CCD}, \mathrm{G}_{16}^{\mathrm{A}}$. It has eight real valued representations.

\begin{tabular}{lrrrrrrrr}
\hline $\mathrm{G}^{\mathrm{PSMS}}\left[\mathrm{G}_{16}^{\mathrm{A}}\right]$ & $E$ & $(12)$ & $(34)$ & $(12)(34)$ & $(56)$ & $(12)(56)$ & $(34)(56)$ & $(12)(34)(56)$ \\
\hline$\Gamma_{1}$ & 1 & 1 & 1 & 1 & 1 & 1 & 1 & 1 \\
$\Gamma_{2}$ & 1 & 1 & 1 & 1 & -1 & -1 & -1 & -1 \\
$\Gamma_{3}$ & 1 & 1 & -1 & -1 & 1 & 1 & -1 & -1 \\
$\Gamma_{4}$ & 1 & 1 & -1 & -1 & -1 & -1 & 1 & 1 \\
$\Gamma_{5}$ & 1 & -1 & 1 & -1 & 1 & -1 & 1 & -1 \\
$\Gamma_{6}$ & 1 & -1 & 1 & -1 & -1 & 1 & -1 & 1 \\
$\Gamma_{7}$ & 1 & -1 & -1 & 1 & 1 & -1 & -1 & 1 \\
$\Gamma_{8}$ & 1 & -1 & -1 & 1 & -1 & 1 & 1 & -1 \\
\hline
\end{tabular}

it is not yet symmetry adapted. Next, we shall apply the symmetry projection operator $\hat{\mathcal{P}}^{\Gamma_{i}}$ - the idempotence Eq. (18) then implies that $\hat{\mathcal{P}}^{\Gamma_{i}} \Psi_{l}$ is automatically an eigenfunction of $\hat{\mathcal{P}}^{\Gamma_{i}}$, because

$$
\hat{\mathcal{P}}^{\Gamma_{i}}\left(\hat{\mathcal{P}}^{\Gamma_{i}} \Psi_{l}\right)=\hat{\mathcal{P}}^{\Gamma_{i}} \Psi_{l},
$$

with eigenvalues 1 or 0 if $\hat{\mathcal{P}}^{\Gamma_{i}} \Psi_{l} \neq 0$ or $\hat{\mathcal{P}}^{\Gamma_{i}} \Psi_{l}=0$, respectively. If $\hat{\mathcal{P}}^{\Gamma_{i}} \Psi_{l} \neq 0$, then we define the symmetry-adapted torsional wavefunction with IREP $\Gamma_{i}$,

$$
\Psi^{\Gamma_{i}}=\mathcal{N}_{i} \hat{\mathcal{P}}^{\Gamma_{i}} \Psi_{l}
$$

where $\mathcal{N}_{i}$ is a normalization constant. The relations we discussed in this Subsection will be important for the later discussion, see Sects. 2.4 and 3.

\subsection{Reduction of the Hamiltonian from full dimensionality to a model with two torsional degrees of freedom}

In order to reduce the Hamiltonian from Eq. (1) from full dimensionality to a model Hamiltonian for the present class of molecules, characterized by two coaxial torsions about the $\mathcal{C}_{2}$ symmetry axis which is oriented along the laboratory $Z$-axis (see items (i) and (ii) in Sect. 1), we employ the approach of Ref. [67]. There, the same type of steps have been demonstrated in order to derive a model Hamiltonian for analogous molecules with a single torsional degree of freedom. The approach will be explained using the model system CCD as an example.

The first step is the familiar Born-Oppenheimer (BO) separation of the electronic and nuclear degrees of freedom [103]. Accordingly, the total wavefunction is written, in general, as a sum of products of electronic wavefunctions $\psi_{\mathrm{e}}(q ; Q)$ depending on the set of electronic (spatial and spin) coordinates $q$, times nuclear wavefunctions $\Psi_{\mathrm{e}, \mathrm{n}}(Q, t)$ depending on the set of nuclear coordinates $Q$ and, in principle, also on time $t$,

$$
\Psi^{\mathrm{mol}}(q, Q, t)=\sum_{\mathrm{e}} \psi_{\mathrm{e}}(q ; Q) \cdot \Psi_{\mathrm{e}, \mathrm{n}}(Q, t)
$$


Note that the symbol $\Psi^{\mathrm{mol}}$ is used here and below for wavefunctions which depend on electronic and nuclear coordinates beyond the Born-Oppenheimer approximation. The sum in Eq. (22) is over electronic states labeled by a set of quantum numbers $e$ which specify the electronic energy, and possibly also complementary properties, e.g. the electronic spin multiplicity, and symmetry. The nuclear wavefunctions carry additional labels $n$ which characterize the initial states; typically, $n$ denotes a set of quantum numbers which specify the rovibronic energies and symmetries. In the present paper, we focus on stationary states, so that the time $t$ will be dropped, for simplicity of the notation; time dependent extensions will be demonstrated in a sequel paper.

The electronic wavefunctions $\psi_{\mathrm{e}}(q ; Q)$ depend parametrically on the nuclear coordinates $Q$. They are obtained as solutions of the electronic Schrödinger equation

$$
\hat{H}_{\mathrm{el}} \psi_{\mathrm{e}}(q ; Q)=V_{\mathrm{e}}(Q) \psi_{\mathrm{e}}(q ; Q),
$$

together with the potential energy surfaces (PES) $V_{\mathrm{e}}(Q)$ depending on $Q$. Here $\hat{H}_{\mathrm{el}}$ is the electronic Hamiltonian - it is the same as the Hamiltonian expressed in Eq. (1), but without the terms for the nuclear kinetic energies. Details of the quantum chemical solution of Eq. (23) will be presented in Sect. 2.3, using the set of coordinates $Q$ which will be specified below. In the present case, we shall focus on electronic singlet states; the electronic quantum numbers $\mathrm{e}=0,1,2$, then label the electronic energies. In particular, the quantum chemistry calculations provide the PES $V_{0}(Q)$ of the electronic ground state. The search for the minimum of $V_{0}(Q)$ yields the minimum structure, also called equilibrium geometry $Q_{\text {eq }}$ of the molecule. For convenience, the value of $V_{0}$ at $Q_{\text {eq }}$ is set equal to zero, $V_{0}\left(Q_{\text {eq }}\right)=0$. As we shall show below, the present molecular symmetry group $\mathrm{G}_{16}^{\mathrm{A}}$ implies that $V_{0}$ actually supports several equivalent minimum structures. The quantum chemistry calculations will be employed to determine just one of these minimum structures (called "the first one", $Q_{\mathrm{eq}_{1}}$ ), together with the PES in a domain around this minimum. The other equilibrium structures $Q_{\mathrm{eq}_{2}}, Q_{\mathrm{eq}_{3}}$, etc. with surrounding domains of the PES will be generated by means of the symmetry operators which have been introduced in Sect. 2.1 The "first" equilibrium structure is used in order to specify the notation for the molecular segments A, B, C and the fragments $i=0,1, \ldots, 6$, and to assign the labels $k$ of the nuclei which belong to these fragments, as shown in Fig. 1. The determination of $V_{0}(Q)$ by means of quantum chemistry and molecular symmetry, and the "first" equilibrium structure $Q_{\mathrm{eq}_{1}}$ with the corresponding fragments, are important results of this first step of the approach; they should be consistent with the criteria (i) and (ii) that defines the present set of molecules, e.g. the equilibrium structure should have a rotational symmetry axis $\mathcal{C}_{2}$ oriented along the laboratory-fixed $Z$-axis. If the equilibrium structure is planar, as for the case of CCD, then for reference, the molecule should be placed in the $X / Z$ plane.

The second step reduces the nuclear Hamiltonian (which describes the nuclear motions within the BO separation of electronic and nuclear dof) from full-d to the degrees of freedom dof of interest - in the present case these are just the two torsional dofs. This step starts from the potential energy surface $V_{0}(Q)$ and the kinetic energy operator

$$
\hat{T}=\hat{T}_{3 N_{n}}=\sum_{i} \sum_{k} \frac{\hat{\vec{P}}_{i k}^{2}}{2 M_{i k}}
$$


of all nuclei; the index $3 N_{n}=78$ for CCD specifies the original total number of nuclear degrees of freedom. At this stage, it is convenient to employ the set $Q$ of all $3 N_{n}$ Cartesian coordinates $\vec{R}_{i k}=\left(X_{i k}, Y_{i k}, Z_{i k}\right)$ in the laboratory frame, together with the nuclear spin variables, with the nuclear center-of-mass as origin and with the $\mathcal{C}_{2}$ symmetry axis oriented along the laboratory-fixed $Z$-axis. The momentum operators are conjugate to these nuclear Cartesian coordinates. According to the approach of Ref. [67], it is then convenient to replace the Cartesian coordinates $\vec{R}_{i k}=\left(X_{i k}, Y_{i k}, Z_{i k}\right)$ of nucleus $k$ in fragment $i$ by cylindrical coordinates $\left(R_{i k}, \Phi_{i k}, Z_{i k}\right)$. Subsequently, more and more of these coordinates, or suitable linear combinations of these coordinates, are frozen in the equilibrium geometry, in a step-by-step manner, corresponding to systematic freezing of those vibrational and rotational dof which will not be considered in the model. For the present simple model CCD, all nuclear coordinates are frozen in the "first" equilibrium structure, except the angles $\Phi_{\mathrm{A}}, \Phi_{\mathrm{B}}, \Phi_{\mathrm{C}}$ describing the internal rotations of fragments A, B, C about the $\mathcal{C}_{2}$ symmetry axis, respectively. The nuclear kinetic energy operator describing these internal molecular rotations is

$$
\hat{T}_{3}=\frac{\hat{L}_{\mathrm{A}}^{2}}{2 I_{\mathrm{A}}}+\frac{\hat{L}_{\mathrm{B}}^{2}}{2 I_{\mathrm{B}}}+\frac{\hat{L}_{\mathrm{C}}^{2}}{2 I_{\mathrm{C}}},
$$

with internal angular momenta $\hat{L}_{S}=-\mathrm{i} \hbar \frac{\partial}{\partial \Phi_{S}}$ for the rotations of the segments $S=$ $\mathrm{A}, \mathrm{B}, \mathrm{C}$ about the $\mathcal{C}_{2}$-axis, and with the corresponding moments of inertia $I_{S}$ which are determined by the "first" equilibrium geometry of the model system.

In the last step, the angles $\Phi_{\mathrm{A}}, \Phi_{\mathrm{B}}$ and $\Phi_{\mathrm{C}}$ are replaced by the two torsional angles $\Phi_{1}=\Phi_{\mathrm{B}}-\Phi_{\mathrm{A}}$ and $\Phi_{2}=\Phi_{\mathrm{C}}-\Phi_{\mathrm{B}}$, and by the angle for overall rotation about the $\mathcal{C}_{2}$-axis, $\Phi=c_{\mathrm{A}} \cdot \Phi_{\mathrm{A}}+c_{\mathrm{B}} \cdot \Phi_{\mathrm{B}}+c_{\mathrm{C}} \cdot \Phi_{\mathrm{C}}$ with coefficients $c_{S}=\frac{I_{S}}{\left(I_{\mathrm{A}}+I_{\mathrm{B}}+I_{\mathrm{C}}\right)}$. Separating the overall rotation, the kinetic energy operator is reduced to

$$
\hat{T}_{2}=\frac{\hat{L}_{1}^{2}}{2 I_{1}}+\frac{\hat{L}_{1} \cdot \hat{L}_{2}}{I_{\mathrm{B}}}+\frac{\hat{L}_{2}^{2}}{2 I_{2}}
$$

with reduced moments of inertia

$$
I_{1}=\frac{I_{\mathrm{A}} \cdot I_{\mathrm{B}}}{I_{\mathrm{A}}+I_{\mathrm{B}}} \quad \text { and } \quad I_{2}=\frac{I_{\mathrm{B}} \cdot I_{\mathrm{C}}}{I_{\mathrm{B}}+I_{\mathrm{C}}} .
$$

The first and last terms of $\hat{T}_{2}$ describe torsions of the neighboring segments A vs. B and B vs. C, respectively. The middle term represents torsional coupling. The derivation of $\hat{T}_{2}$ from $\hat{T}_{3}$ is entirely analogous to the derivation of the kinetic energy operator of the familiar Thiele-Wilson Hamiltonian [104] for the coupled vibrations of bonds $\mathrm{AB}$ and $\mathrm{BC}$ of a triatomic molecule $\mathrm{ABC}$. Finally, neglecting nonadiabatic and dipole couplings, the $2 \mathrm{~d}$ model Hamiltonians describing adiabatic torsions along $\Phi_{1}$ and $\Phi_{2}$ in electronic states $e$ are written as

$$
\hat{H}_{2 e}\left(\Phi_{1}, \Phi_{2}\right)=\hat{T}_{2}+V_{\mathrm{e}}\left(\Phi_{1}, \Phi_{2}\right)
$$

with the PES depending explicitly on the torsional angles $\Phi_{1}$ and $\Phi_{2}$, while all other dof are frozen in the "first" equilibrium geometry. Note, that this derivation of the nuclear 
Hamiltonian started from all nuclear Cartesian coordinates in the laboratory frame, whereas it ends with the expression in terms of the molecule-fixed torsional angles, analogous to Ref. [67].

The derivation allows various extensions. For example, one may include additional vibrational degrees of freedom [67]. If the molecule interacts with a linearly $Z$-polarized laser field $E(t)=\left(0,0, E_{Z}(t)\right)$, the Hamiltonian is written in dipole approximation as

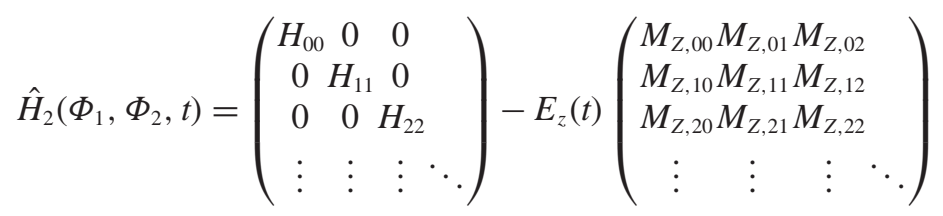

where $M_{Z, e e}=M_{Z, e e}\left(\Phi_{1}, \Phi_{2}\right)$ are the $Z$-components of the dipole operators depending on the torsional angles, whereas $M_{Z, e e^{\prime}}=M_{Z, e e^{\prime}}\left(\Phi_{1}, \Phi_{2}\right)$ are the corresponding dipole transition matrix elements for transitions between states $e$ and $e^{\prime}$, respectively. The model Hamiltonian may also be extended by nonadiabatic couplings $[67,71]$.

\subsection{The quantum chemistry of CCD and the method for calculation of a localized torsional wavefunction}

To calculate the PES along the two torsional degrees of freedom, an active space with 12 electrons in 10 orbitals $(12,10)$ was chosen such that all $\pi$ orbitals of the molecule are included, that are relevant for the singlet ground and two lowest excited states of $\pi \pi^{*}$ character. This chosen reduced active space, compared to the full $\pi$-system $(18,16)$, is the best compromise between computational efforts and a precise description of all relevant excitations. With this active space, a complete active space selfconsistent field (CASSCF) [105] calculation is performed, using the cc-pVDZ [106] basis set.

Firstly, a geometry optimization for the electronic ground state is performed to locate the ground state equilibrium conformation. This calculation is done using the program package PC GAMESS [107,108].

Based on this equilibrium geometry, a scan of the two-dimensional PES of the three lowest singlet states is performed using the State-Averaged CASSCF method by varying the torsional angle $\Phi_{1}$ between $-\frac{\pi}{2}$ and $\frac{\pi}{2}$ and the torsional angle $\Phi_{2}$ between 0 and $\frac{\pi}{2}$ with a step size of $0.028 \cdot \pi$, (i.e. $5^{\circ}$ ), while keeping the other dof frozen. The consistency of the active space along both coordinates, $\Phi_{1}$ and $\Phi_{2}$, is ensured by carefully selecting fully converged CASSCF wavefunctions of previous steps as an initial guess and by close inspection of several one-electron properties like nonadiabatic couplings and transition dipole moments.

From this data, the full two-dimensional PES describing a full $2 \pi$ torsion along both angles is constructed following the symmetry operations described in Sect. 2 and illustrated in Fig. 3. All CASSCF calculations were carried out with the Molpro2010 program package [109].

The torsional eigenfunctions in the electronic ground state are calculated by directly diagonalizing $\hat{H}_{2 e}\left(\Phi_{1}, \Phi_{2}\right)$, Eq. (28), using the Fourier Grid Hamiltonian method [110] 


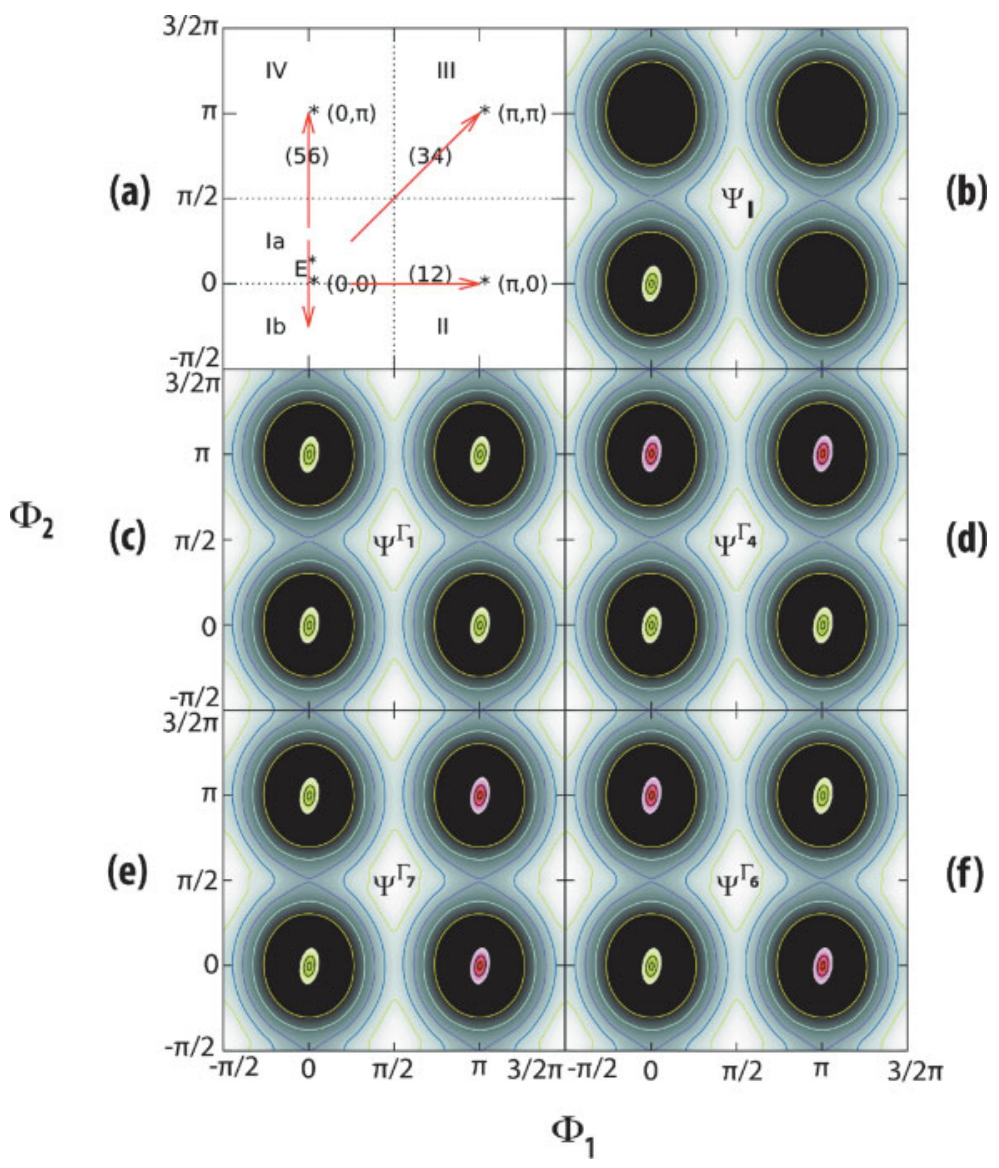

Fig. 3. Potential energy surface and torsional wavefunctions of CCD and four NSIs of CCD in the electronic ground state, vs. torsional angles $\Phi_{1}$ and $\Phi_{2}$. All other degrees of freedom of CCD are frozen in the "first" equilibrium structure of $\operatorname{CCD}\left(\Phi_{1, \mathrm{eq}_{1}}, \Phi_{2, \mathrm{eq}}\right)$, abbreviated as $(0,0)$, see Fig. 1. (a) Scheme for generation of three equivalent equilibrium structures $(0, \pi),(\pi, 0),(\pi, \pi)$ of CCD by application of the generators (12), (56), (34) of the molecular symmetry group $\mathrm{G}_{16}^{\mathrm{A}}$ to $(0,0)$, and scheme for generating the potential energy surface $V_{0}\left(\Phi_{1}, \Phi_{2}\right)$ in the domains $I b, I I, I I I, I V$ by application of the generators $E^{*}$ and subsequently (12), (34) and (56) to $V_{0}\left(\Phi_{1}, \Phi_{2}\right)$ in domain $I a$ and in domain $I=I a$ and $I b$, respectively. (b) Contour plots of $V_{0}\left(\Phi_{1}, \Phi_{2}\right)$ and the torsional wavefunction $\Psi_{l}\left(\Phi_{1}, \Phi_{2}\right)$ localized in the potential well close to $(0,0)$. (c)-(f): Molecular symmetry adapted torsional wavefunctions $\Psi^{\Gamma_{1}}\left(\Phi_{1}, \Phi_{2}\right)$, $\Psi^{\Gamma_{4}}\left(\Phi_{1}, \Phi_{2}\right), \Psi^{\Gamma_{7}}\left(\Phi_{1}, \Phi_{2}\right), \Psi^{\Gamma_{6}}\left(\Phi_{1}, \Phi_{2}\right)$ embedded in $V_{0}\left(\Phi_{1}, \Phi_{2}\right)$, respectively. Potential contours are drawn for $V_{0}\left(\Phi_{1}, \Phi_{2}\right)=(0.5,1,1.5,2,2.5,3) \mathrm{eV}$. Contours of the wavefunctions are drawn for $\pm 0.9, \pm 0.5$ and \pm 0.1 times the maximum absolute values of symmetry adapted wavefunctions. Positive and negative values of the wavefunctions are indicated by green and red colors, respectively.

for the domain of the single potential energy well around the "first" equilibrium structure, within the range of $-\frac{\pi}{2}$ to $\frac{\pi}{2}$ for both torsional angles $\Phi_{1}$ and $\Phi_{2}$. For each degree of freedom a grid of 256 points for each dof was chosen. 


\subsection{The NSIs of CCD}

To identify the NSIs of molecules several techniques are necessary: the symmetrization postulate [8], the permutation subgroup of the molecular symmetry group [96,99] and the nuclear spin hypothesis [111], see also [49]. We will derive the conditions which every molecular state must agree with to fulfill the symmetrization postulate, i.e. we find the irreducible molecular representation $\Gamma^{\mathrm{mol}}$. Afterwards, the symmetry of the nuclear spin states and the torsional states of CCD within the molecular symmetry group are found. This Subsection closes with determining the NSIs of CCD.

According to the symmetrization postulate every state of a quantum system must hold its sign if a pair of identical bosons is exchanged, and must change its sign if a pair of identical fermions is exchanged once. To apply this theorem the spin quantum numbers of the nuclei which are exchanged have to be known. For CCD, we have three types of nuclei, ${ }^{1} \mathrm{H},{ }^{12} \mathrm{C},{ }^{16} \mathrm{O}$, which carry the spins $\frac{1}{2}, 0,0$, respectively. Consequently, the nuclei ${ }^{12} \mathrm{C}$ and ${ }^{16} \mathrm{O}$ are bosons, since they carry an integer spin; the nuclei ${ }^{1} \mathrm{H}$ are fermions, they carry half-integer spin. Since the symmetrization postulate states nothing about permutation-inversions, the group which is relevant for the identification of the NSIs of $\mathrm{CCD}$ is the permutation subgroup of $\mathrm{G}_{16}^{\mathrm{A}}$, i.e. the group $\mathrm{G}^{\mathrm{PSMS}}\left[\mathrm{G}_{16}^{\mathrm{A}}\right]$, see Eq. (8) [49]. Some of the consequences for the identification of the NSIs, when limiting the number of permutations to those which are feasible, are discussed in Sect. 4.1 of Ref. [49]. The influence of the field which is used to orient the molecule on the symmetry of the system is not trivial. It will be discussed in detail in a subsequent publication, see also Sect. 5.5 of Ref. [49]. Here, it suffices to say that the results of this section will remain valid.

To find $\Gamma^{\mathrm{mol}}$, we need only to consider the transformation properties of the system's wavefunctions under the generators of $\mathrm{G}^{\mathrm{PSMS}}\left[\mathrm{G}_{16}^{\mathrm{A}}\right]$. For CCD these are the operations (12), (34) and (56). Thus

\begin{tabular}{l|lll}
$\mathcal{R}$ & $(12)$ & $(34)$ & $(56)$ \\
\hline$\Psi^{\mathrm{mol}}$ & $(-1) \cdot \Psi^{\mathrm{mol}}$ & $(+1) \cdot \Psi^{\mathrm{mol}}$ & $(+1) \cdot \Psi^{\mathrm{mol}}$
\end{tabular}

since (12) permutes an odd number of pairs of fermionic nuclei, and (34) and (56) permute even numbers of pairs of fermionic nuclei. The characters $-1,1,1$ of the generators (12), (34), (56) then yield the irreducible representation

$$
\Gamma^{\mathrm{mol}}=\Gamma_{5}
$$

for the present class of molecules, compare with Table 4.

For defining a NSI of a molecule based on the conditions from Eq. (30), the nuclear spin hypothesis is used [111]. This hypothesis states that for any molecular state it is appropriate to write

$$
\Psi^{\mathrm{mol}}=\psi_{\mathrm{e}} \cdot \Psi_{\mathrm{e}, \mathrm{n}} \approx \Psi^{\mathrm{rve}} \cdot \Psi^{\mathrm{nu} . \mathrm{sp}}
$$

where $\Psi^{\text {rve }}$ denotes the rovibronic wavefunction and $\Psi^{\text {nu.sp }}$ labels the wavefunctions for the nuclear spins. Different combinations of wavefunctions $\Psi^{\text {rve }}$ and $\Psi^{\text {nu.sp }}$ with the product of the two transforming according to $\Gamma^{\mathrm{mol}}$ are defined as different NSIs. Obviously, this assumption is an approximation - on very long time scales NSIs are not 
stable species of a chemical compound, due to intramolecular interactions of nuclear spins. However, at least for closed shell molecules, the time-scale of interconversion of NSIs is much slower than the torsional dynamics, which shall be investigated here $[8,10]$.

How the symmetry of the nuclear spin states of a molecule can be found with the help of the molecular symmetry group is well described in the literature [99]. To find the representation which is spanned by the entity of all nuclear spin states, one has to count the number of spin states which are invariant under a given operation of the group $\mathrm{G}^{\mathrm{PSMS}}\left[\mathrm{G}_{16}^{\mathrm{A}}\right]$. They give the character of the representation $\Gamma^{\text {nu.sp }}$ for the operation in question. CCD, for example, has in total $2^{10}=1024$ spin states - each proton contributes 2 states, all other nuclei do not posses any nuclear spin. If one applies the operation (12) to these states, all states of fragment 3 to 6 remain unchanged, as well as the $\alpha \alpha$ and $\beta \beta$ states of the oxo-ring. Only the states $\alpha \beta$ and $\beta \alpha$ are interchanged. Thus, $2 \cdot 2^{8}=\overline{2^{9}}=512$ states are invariant under the operation (12) which gives the character of the reducible representation spanned by all spin states. Proceeding analogously for the remaining spin states one obtains

\begin{tabular}{l|llllllll}
$\mathcal{R}$ & $(1)$ & $(12)$ & $(34)$ & $(56)$ & $(12)(34)$ & $(12)(56)$ & $(34)(56)$ & $(12)(34)(56)$ \\
\hline$\Gamma^{\text {nu.sp. }}$ & 1024 & 512 & 256 & 256 & 128 & 128 & 64 & 32
\end{tabular}.

Reducing this representation into its irreducible components gives

$$
\begin{aligned}
\Gamma^{\text {nu.sp. }}= & 300 \cdot \Gamma_{1} \oplus 180 \cdot \Gamma_{2} \oplus 180 \cdot \Gamma_{3} \oplus 108 \cdot \Gamma_{4} \oplus \\
& \oplus 100 \cdot \Gamma_{5} \oplus 60 \cdot \Gamma_{6} \oplus 60 \cdot \Gamma_{7} \oplus 36 \cdot \Gamma_{8} .
\end{aligned}
$$

The result Eq. (34) provides the answer to another question which has been asked in the Introduction, i.e. how to separate the 1024 nuclear spin eigenfunctions of CCD into sets which can be assigned to its eight NSIs, characterized by the IREPs $\Gamma_{1}-\Gamma_{8}$. Next, these nuclear spin states have to be combined with the torsional states to fulfill Eq. (31).

Before the correct torsional states are identified, a few aspects about the rotational and the vibronic states are worth mentioning. In the electronic ground state CCD is a rigid molecule, i.e. the time scales of any contorsional motion are much longer than the time scales on which molecular events typically take place. As a consequence, it is appropriate to write any molecular state in the electronic ground state in the form

$$
\Psi^{\mathrm{mol}} \approx \Psi^{\mathrm{rot}} \cdot \Psi^{\mathrm{tor}} \cdot \Psi^{\mathrm{vib}} \cdot \Psi^{\text {nu.sp. }} \cdot \psi^{\mathrm{elec}}
$$

and to assume that the rotational motions can be manipulated separately from the torsional motion. In Eq. (35) $\Psi^{\text {rot }}$ denotes the wavefunction for the rotational motions, $\Psi^{\mathrm{vib}}$ the wavefunction for the vibrational motions except the torsions, $\psi^{\text {elec }}$ the wavefunction for the electrons and $\Psi^{\text {nu.sp. }}$ the wavefunction for the nuclear spins. Here, it is assumed that the translational motion of the molecule is separated from internal motions. Some authors call the molecular wavefunction excluding the translational motions internal wavefunction [99]; this distinction will be omitted in this paper. If the temperature before the interaction with the laser pulse used to orient the molecule is so low that basically only the lowest rotational state is populated and, additionally, the vibronic ground 
state is non-degenerate

$$
\Psi^{\mathrm{rot}} \cdot \Psi^{\mathrm{vib}} \cdot \psi^{\mathrm{elec}} \sim \Gamma_{1}
$$

Using this, different NSIs are identified by

$$
\Gamma^{\mathrm{tor}} \otimes \Gamma^{\mathrm{nu} . \mathrm{sp} .} \stackrel{!}{=} \Gamma^{\mathrm{mol}}
$$

different combinations of $\Gamma^{\text {tor }}$ and $\Gamma^{\text {nu.sp }}$ fulfilling this equation represent different NSIs.

To find the symmetry of the torsional states it is sufficient to find the symmetries of the basis functions

$$
\begin{aligned}
\Psi_{k_{\Phi_{1}}, k_{\Phi_{2}}}\left(\Phi_{1}, \Phi_{2}\right) & =\Psi_{k_{\Phi_{1}}}\left(\Phi_{1}\right) \cdot \Psi_{k_{\Phi_{2}}}\left(\Phi_{2}\right) \\
& =\frac{1}{2 \pi} \exp \left(\mathrm{i} k_{\Phi_{1}} \Phi_{1}\right) \exp \left(\mathrm{i} k_{\Phi_{2}} \Phi_{2}\right),
\end{aligned}
$$

since: (i) the true eigenfunctions can always be written as linear combinations of basis functions of type Eq. (38), no matter how reasonable this ansatz is; (ii) exact eigenfunctions can be composed exclusively of basis functions of the same symmetry [112]. The symmetry of the functions Eq. (38) in the group $\mathrm{G}^{\mathrm{PSMS}}\left[\mathrm{G}_{16}^{\mathrm{A}}\right]$ can be found once the transformation properties of the angles $\Phi_{1}$ and $\Phi_{2}$ under the generators (12), (34) and (56) are known. The angles $\Phi_{1}$ and $\Phi_{2}$ transform under these operations as

\begin{tabular}{l|lcl}
$\mathcal{R}$ & $(12)$ & $(34)$ & $(56)$ \\
\hline$\Phi_{1}$ & $\Phi_{1}+\pi$ & $\Phi_{1}-\pi$ & $\Phi_{1}$ \\
$\Phi_{2}$ & $\Phi_{2}$ & $\Phi_{2}+\pi$ & $\Phi_{2}-\pi$
\end{tabular}

and therefore

\begin{tabular}{c|ccc}
$\mathcal{R}$ & $(12)$ & $(34)$ & $(56)$ \\
\hline$\Psi_{k_{\Phi_{1}}}$ & $(-1)^{k \Phi_{1}} \cdot \Psi_{k_{\Phi_{1}}}$ & $(-1)^{k \Phi_{1}} \cdot \Psi_{k_{\Phi_{1}}}$ & $\Psi_{k_{\Phi_{1}}}$ \\
$\Psi_{k_{\Phi_{2}}}$ & $\Psi_{k_{\Phi_{2}}}$ & $(-1)^{k \Phi_{2}} \cdot \Psi_{k_{\Phi_{2}}}$ & $(-1)^{k \Phi_{2}} \cdot \Psi_{k_{\Phi_{2}}}$
\end{tabular}.

Now one can conclude that

$$
\Psi_{k_{\Phi_{1}}, k_{\Phi_{2}}}\left(\Phi_{1}, \Phi_{2}\right) \sim\left\{\begin{array}{lllllll}
\Gamma_{1} & \text { if } & k_{\Phi_{1}} & \text { even } & \text { and } & k_{\Phi_{2}} & \text { even } \\
\Gamma_{4} & \text { if } & k_{\Phi_{1}} & \text { even } & \text { and } & k_{\Phi_{2}} & \text { odd } \\
\Gamma_{7} & \text { if } & k_{\Phi_{1}} & \text { odd } & \text { and } & k_{\Phi_{2}} & \text { even } \\
\Gamma_{6} & \text { if } & k_{\Phi_{1}} & \text { odd } & \text { and } & k_{\Phi_{2}} & \text { odd }
\end{array} .\right.
$$

The result Eq. (41) provides the answer to still another question which has been asked in the Introduction, i.e. four $\left(\Gamma_{1}, \Gamma_{4}, \Gamma_{6}, \Gamma_{7}\right)$ out of eight $\left(\Gamma_{1}-\Gamma_{8}\right)$ NSIs "survive" as $T \rightarrow 0$.

In the last step, the torsional eigenstates with IREPs $\Gamma_{1}, \Gamma_{4}, \Gamma_{6}, \Gamma_{7}$ have to be combined with the nuclear spin states from the previous section to fulfill Eqs. (30). The correct combinations of torsional and nuclear spin states can be easily found with the 
help of the direct product table of $\mathrm{G}^{\mathrm{PSMS}}\left[\mathrm{G}_{16}^{\mathrm{A}}\right]$, which is obtained from the $8 \times 8$ entries in the top left of Table 3, without the labels "+". For CCD one obtains the four NSIs $\Gamma^{\text {tor }}\left[\Gamma^{\text {nu.sp. }}\right]$

$$
\begin{array}{ll}
\Gamma_{1}\left[\Gamma_{5}\right] & \text { (100 nuclear spin states) } \\
\Gamma_{4}\left[\Gamma_{8}\right] & \text { (36 nuclear spin states) } \\
\Gamma_{7}\left[\Gamma_{3}\right] & \text { (180 nuclear spin states) } \\
\Gamma_{6}\left[\Gamma_{2}\right] & \text { (180 nuclear spin states) }
\end{array}
$$

where the number of spin states are obtained from Eq. (34).

For later investigations, the symmetry of the torsional states of each nuclear spin isomer in the full MS group will be interesting as well. In particular, the parity of the torsional states is important, i.e. their transformation properties under the fourth generator $E^{*}$. For the two torsional angles, $\Phi_{1}$ and $\Phi_{2}$, it holds that

\begin{tabular}{l|l}
$\mathcal{R}$ & $E^{*}$ \\
\hline$\Phi_{1}$ & $-\Phi_{1}$ \\
$\Phi_{2}$ & $-\Phi_{2}$
\end{tabular}

i.e. $E^{*}$ transforms every basis function, Eq. (38), into its complex conjugate $\Psi_{-k_{\phi_{1}},-k_{\Phi_{2}}}$. Thus, the parity adapted eigenfunctions must be real linear combinations of $\Psi_{k_{\Phi_{1}}, k_{\Phi_{2}}}$ and $\Psi_{-k_{\Phi_{1}},-k_{\Phi_{2}}}$. Two real linear combinations can be found

$$
\begin{aligned}
& \Psi_{k_{\phi_{1}}, k_{\Phi_{2}}}^{+} \equiv \frac{1}{\sqrt{2}}\left(\Psi_{k_{\phi_{1}}, k_{\phi_{2}}}+\Psi_{-k_{\phi_{1}},-k \phi_{\phi_{2}}}\right) \\
& \Psi_{k_{\phi_{1}}, k \phi_{2}}^{-} \equiv \frac{\mathrm{i}}{\sqrt{2}}\left(\Psi_{k_{\phi_{1}}, k_{\phi_{2}}}-\Psi_{-k_{\phi_{1}},-k \phi_{\Phi_{2}}}\right)
\end{aligned}
$$

with $\Psi_{k_{\phi_{1}}, k_{\Phi_{2}}}^{+}$having even parity and $\Psi_{k_{\phi_{1}}, k_{\Phi_{2}}}^{-}$having odd parity. Since we can form these combinations for every pair of quantum numbers $k_{\phi_{1}}$ and $k_{\phi_{2}}$ each NSI can have torsional states with either even or odd parity. We will illustrate this further in Sect. 3.5.

\section{Results and discussion}

This section is subdivided into five subsections. All of them present and discuss results for the model system CCD which has been specified in Sects. 1, 2.1 and 2.2 as representative of the new class of molecules which satisfy the criteria (i), (ii) given in the Introduction. Sect. 3.1 summarizes those results of Sect. 2.4 which are relevant for the rest of this section. The other four subsections present and discuss specific results which are obtained by means of the methods presented in Sect. 2.3 combined with Sect. 2.4. Specifically, Sect. 3.2 has the quantum chemical results for the "first" equilibrium structure, as well as for the potential energy surface $V_{0}\left(\Phi_{1}, \Phi_{2}\right)$ in a neighboring domain, with restricted values of the two torsional angles. Sect. 3.3 employs molecular symmetry to generate the complete set of equivalent minimum structures, and to extend $V_{0}\left(\Phi_{1}, \Phi_{2}\right)$ from a restricted range to all values of the torsional angles. Sect. 3.4 
presents a torsional wavefunction which is localized in the single well of the potential energy surface in the domain close to the "first" equilibrium structure. Sect. 3.5 applies molecular symmetry projection operators to that "localized" wavefunction in order to generate molecular symmetry adapted torsional eigenfunctions for the different NSIs of CCD. Atomic units are employed throughout this section, unless stated otherwise.

\subsection{Results of molecular symmetry}

As shown in Sect. 2.4, CCD supports eight NSIs. Each one of them is characterized by a specific IREP of the subgroup $\mathrm{G}^{\mathrm{PSMS}}\left[\mathrm{G}_{16}^{\mathrm{A}}\right]$ of the molecular symmetry group $\mathrm{G}_{16}^{\mathrm{A}}$, $c f$. Table 4, for the symmetry-adapted nuclear spin wavefunction. Here we focus on the subset of four NSIs with IREPs $\Gamma_{5}, \Gamma_{8}, \Gamma_{3}$ and $\Gamma_{2}$, see Eqs. (41), respectively, which "survive" as $T \rightarrow 0$. These selected nuclear spin wavefunctions are multiplied by torsional wavefunctions with specific IREPs $\Gamma_{1}, \Gamma_{4}, \Gamma_{7}, \Gamma_{6}$, see again Eqs. (41), respectively. In the following subsections, we shall determine the energetically lowest quadruplet of those torsional eigenfunctions.

It is remarkable that this subset of four NSIs at $T \rightarrow 0$ contains just half of the eight NSIs which are available for CCD. This is reminiscent of Heisenberg's and Hund's example of molecular hydrogen, where $\mathrm{p}-\mathrm{H}_{2}$ "survives" at $T \rightarrow 0$, i.e. just one out of two NSIs of hydrogen.

\subsection{The "first" equilibrium structure of CCD, and the potential energy surface $V_{0}$ in a neighboring domain}

The quantum chemistry calculations Sect. 2.3 yield the "first" equilibrium structure of $\mathrm{CCD}$, as shown in the top panel of Fig. 1. Its molecular point group is $\mathrm{C}_{2 \mathrm{v}}$, i.e. it is planar, and it has a $\mathcal{C}_{2}$ symmetry axis. We assume that this $\mathcal{C}_{2}$-axis has been oriented along the laboratory $Z$-axis, $c f$. the discussions in Sects. 1 and 2.1. This "first" equilibrium structure will be called $Q_{\mathrm{eq}_{1}}$. It is used to specify the notations, see the figure legend 1. For example, the two torsional angles describing torsions of fragments $\mathrm{B} v s$. A and C vs. B are set to $\Phi_{1, \mathrm{eq}_{1}}=0$ and $\Phi_{2, \mathrm{eq}_{1}}=0$, respectively. The subsequent results are obtained by "freezing" all degrees of freedom at the values for $Q_{\mathrm{eq}_{1}}$, except the two torsional angles $\Phi_{1}$ and $\Phi_{2}$. The values of the corresponding torsional moments of inertia are $I_{1}=2.21 \cdot 10^{5} m_{\mathrm{e}} \cdot a_{0}^{2}$ and $I_{2}=2.36 \cdot 10^{5} m_{\mathrm{e}} \cdot a_{0}^{2}$, respectively. These are obtained from the moments of inertia at the "first" equilibrium structure, $I_{\mathrm{A}}=3.47 \cdot 10^{5} \mathrm{~m}_{\mathrm{e}} \cdot a_{0}^{2}$, $I_{\mathrm{B}}=6.07 \cdot 10^{5} \mathrm{~m}_{\mathrm{e}} \cdot a_{0}^{2}$, and $I_{\mathrm{C}}=3.86 \cdot 10^{5} \mathrm{~m}_{\mathrm{e}} \cdot a_{0}^{2}$ for intramolecular rotations of the fragments $\mathrm{A}, \mathrm{B}$, and $\mathrm{C}$ around the axis $\mathcal{C}_{2}$, respectively, using Eq. (27).

The quantum chemistry calculations also yield the PES $V_{\mathrm{e}}\left(\Phi_{1}, \Phi_{2}\right)$, as explained in Sect. 2.3. Here we focus on the PES $V_{0}$ for the electronic ground state. It is efficient to carry out those rather expensive evaluations of $V_{0}\left(\Phi_{1}, \Phi_{2}\right)$ in a domain which is as small as possible, close to $Q_{\mathrm{eq}_{1}}$, and to generate the rest of $V_{0}\left(\Phi_{1}, \Phi_{2}\right)$ in complementary domains, by means of operations based on molecular symmetry. For this purpose, we define the following domains, $c f$. Fig. 3a:

- domain Ia: $-\frac{\pi}{2} \leq \Phi_{1}<\frac{\pi}{2}, 0 \leq \Phi_{2}<\frac{\pi}{2}$ 
- domain Ib: $-\frac{\pi}{2} \leq \Phi_{1}<\frac{\pi}{2},-\frac{\pi}{2} \leq \Phi_{2}<0$

- domain I: $-\frac{\pi}{2} \leq \Phi_{1}<\frac{\pi}{2},-\frac{\pi}{2} \leq \Phi_{2}<\frac{\pi}{2}$

- domain II: $\frac{\pi}{2} \leq \Phi_{1}<\frac{3 \pi}{2},-\frac{\pi}{2} \leq \Phi_{2}<\frac{\pi}{2}$

- domain III: $-\frac{\pi}{2} \leq \Phi_{1}<\frac{\pi}{2}, \frac{\pi}{2} \leq \Phi_{2}<\frac{3 \pi}{2}$

- domain IV: $\frac{\pi}{2} \leq \Phi_{1}<\frac{3 \pi}{2}, \frac{\pi}{2} \leq \Phi_{2}<\frac{3 \pi}{2}$

Note that domain I consists of domains Ia plus Ib, surrounding the "first" equilibrium structure $Q_{\text {eq } 1}$.

In practice, the quantum chemistry calculations are carried out just in the "first" domain Ia, neighboring to $Q_{\text {eq } 1}$. The PES $V_{0}\left(\Phi_{1}, \Phi_{2}\right)$ in domain Ia is included in Fig. $3 b$.

\subsection{Generation of all equivalent equilibrium structures of $\mathrm{CCD}$, and of the PES $V_{0}$ for arbitrary torsional angles}

The complete set of all equivalent equilibrium structures of CCD may be generated by applying the molecular symmetry operators $E,(12),(34), \ldots,(12)(34)(56)^{*}$ of the molecular symmetry group $\mathrm{G}_{16}^{\mathrm{A}}$ to the "first" equilibrium structure $Q_{\text {eq }}, c f$. Eqs. (11) and (12). Due to the transformation properties of the two torsional angles, see Eqs. (39) and (46), CCD has altogether four equivalent equilibrium structures which are characterized by the torsional angles $\left(\Phi_{1, \text { eq }}, \Phi_{2, \mathrm{eq}_{l}}\right)=(0,0),(0, \pi),(\pi, 0)$ and $(\pi, \pi)$ for $l=1,2,3,4$, respectively, see Fig. 3a. Apparently, the set of equilibrium structures $(0, \pi),(\pi, 0)$ and $(\pi, \pi)$ can be generated by a applying just the generators (12), (56) and (34) of $\mathrm{G}_{16}^{\mathrm{A}}$ to $(0,0)$.

Likewise, the potential energy surface $V_{0}\left(\Phi_{1}, \Phi_{2}\right)$ in domain Ib is obtained by application of the generator $E^{*}$ of $\mathrm{G}_{16}^{\mathrm{A}}$, i.e. inversion $E^{*}$ of $V_{0}\left(\Phi_{1}, \Phi_{2}\right)$ in domain Ia, see Fig. 3a. This provides $V_{0}\left(\Phi_{1}, \Phi_{2}\right)$ in the domain I which surrounds the "first" equilibrium structure $Q_{\text {eq }}$. Subsequent applications of the other generators (12), (56), (34) of $\mathrm{G}_{16}^{\mathrm{A}}$ to $V_{0}\left(\Phi_{1}, \Phi_{2}\right)$ in domain I provide $V_{0}\left(\Phi_{1}, \Phi_{2}\right)$ in the domains II, IV and III surrounding the equilibrium structures $(0, \pi),(\pi, 0)$ and $(\pi, \pi)$, respectively, $c f$. Fig. 3a.

The resulting $V_{0}\left(\Phi_{1}, \Phi_{2}\right)$ for arbitrary torsional angles is illustrated in Fig. 3b. It shows four equivalent potential wells which are centered at the equilibrium structures $(0,0)-(\pi, \pi)$ and separated from each other by potential barriers. Note that the potential barriers for torsions along $\Phi_{1}$ are higher than those for torsions along $\Phi_{2}$. The potential has cyclic boundaries for the torsions along $\Phi_{1}$ or $\Phi_{2}$, allowing cyclic extensions of $V_{0}\left(\Phi_{1}, \Phi_{2}\right)$ to arbitrary torsional angles, even beyond the domain shown in Fig. 3b. The molecular symmetry group $\mathrm{G}_{16}^{\mathrm{A}}$ implies that $V_{0}\left(\Phi_{1}, \Phi_{2}\right)$ is invariant with respect to applications of the generators (12), (34), (56) and $E^{*}$, corresponding to the mappings $V_{0}\left(\Phi_{1}, \Phi_{2}\right) \rightarrow V_{0}\left(\Phi_{1}+\pi, \Phi_{2}\right), V_{0}\left(\Phi_{1}+\pi, \Phi_{2}+\pi\right), V_{0}\left(\Phi_{1}, \Phi_{2}+\pi\right)$ and $V_{0}\left(-\Phi_{1},-\Phi_{2}\right)$, respectively. On first glance, Fig. 3b may suggest additional symmetries, e.g. the abscissa and ordinates $\left(\Phi_{2}=0\right.$ and $\left.\Phi_{1}=0\right)$ appear as symmetry lines for reflection, but close inspection reveals that $V_{0}\left(\Phi_{1}, \Phi_{2}\right) \neq V_{0}\left(-\Phi_{1}, \Phi_{2}\right)$ and $V_{0}\left(\Phi_{1}, \Phi_{2}\right)$ $\neq V_{0}\left(\Phi_{1},-\Phi_{2}\right)$. In fact, the molecular symmetry group $\mathrm{G}_{16}$ does not contain any symmetry operators which correspond to the hypothetical mappings $\left(\Phi_{1}, \Phi_{2}\right) \rightarrow\left(-\Phi_{1}, \Phi_{2}\right)$ or $\rightarrow\left(\Phi_{1},-\Phi_{2}\right)$, respectively. 


\subsection{The torsional wavefunction localized close to the "first" equilibrium structure}

Figure $3 \mathrm{~b}$ shows the "localized" torsional wavefunction $\Psi_{l}\left(\Phi_{1}, \Phi_{2}\right)$ embedded in the potential $V_{0}\left(\Phi_{1}, \Phi_{2}\right)$ which has been computed for the domain I surrounding the "first" equilibrium structure $(0,0)$, by means of the Fourier Grid Hamiltonian method [110], as outlined in Sect. 2.3. Apparently, $\Psi_{l}\left(\Phi_{1}, \Phi_{2}\right)$ has ellipsoidal shape. The modest rotation of the ellipsoidal axes with respect to the abscissa $\left(\Phi_{2}=0\right)$ and ordinate $\left(\Phi_{1}=0\right)$ is due to the torsional coupling term of the CCD model Hamiltonian, $c f$. Eqs. (26) and (28).

\subsection{2d molecular symmetry adapted torsional wavefunctions for NSI of CCD}

The symmetry projection operators $\hat{P}^{\Gamma_{l}}, l=1,4,7,6$ (see Eq. 19) of the permutation subgroup $\mathrm{G}^{\mathrm{PSMS}}\left[\mathrm{G}_{16}^{\mathrm{A}}\right]$ of $\mathrm{G}_{16}^{\mathrm{A}}$ operating on the "localized" torsional wavefunction $\Psi_{l}\left(\Phi_{1}, \Phi_{2}\right)$ (Fig. 3b) generate the symmetry-adapted torsional wavefunctions (Eq. 21) with IREPs $\Gamma_{1}, \Gamma_{7}, \Gamma_{4}, \Gamma_{6}$, respectively. These wavefunctions $\Psi^{\Gamma_{l}}\left(\Phi_{1}, \Phi_{2}\right), l=1,7,4,6$ multiply the nuclear spin functions for the NSIs of CCD, with selective IREPs $\Gamma_{5}, \Gamma_{3}$, $\Gamma_{8}, \Gamma_{2}, c f$. Eqs. (41) and (42). They are illustrated in Figs. 3c, 3d, 3e and 3f, respectively, in the order of increasing torsional energies. Apparently, the quadruplet of torsional wavefunctions shown in Figs. $3 \mathrm{c}-3 \mathrm{f}$ consists of four "torsional" lobes which are located close to the equilibrium structures $(0,0),(0, \pi),(\pi, 0)$, and $(\pi, \pi)$. By construction, i.e. due to the generation of these lobes by means of the symmetry projection operators Eq. (19), the four torsional lobes all have the same ellipsoidal shapes and orientations of their ellipsoidal axes, but the signs of the torsional lobes may differ, indicating nodal lines between the lobes with opposite signs.

From their nodal patterns we deduce that $\Psi^{\Gamma_{1}}\left(\Phi_{1}, \Phi_{2}\right)$, which has zero nodes, represents the torsional ground state. The torsional wavefunctions $\Psi^{\Gamma_{4}}\left(\Phi_{1}, \Phi_{2}\right)$ and $\Psi^{\Gamma_{7}}\left(\Phi_{1}, \Phi_{2}\right)$ have nodal lines essentially parallel to the abscissa $\left(\Phi_{2}=0\right)$ and the ordinate $\left(\Phi_{1}=0\right)$, indicating that they represent the next higher states for torsions along $\Phi_{1}$ and $\Phi_{2}$, respectively. Accordingly, one can consider $\Psi^{\Gamma_{1}}\left(\Phi_{1}, \Phi_{2}\right)$ and $\Psi^{\Gamma_{4}}\left(\Phi_{1}, \Phi_{2}\right)$ as the energetically lowest doublet of states for torsion along $\Phi_{1}$, whereas $\Psi^{\Gamma_{1}}$ and $\Psi^{\Gamma_{7}}$ represent the corresponding doublet for torsion along angle $\Phi_{2}$. Since the barrier height for tunneling along $\Phi_{1}$ is higher than for tunneling along $\Phi_{2}$, and since the values of the corresponding torsional moments of inertia $I_{1}$ and $I_{2}$ are about equal, it follows that the tunneling times are longer and the tunneling splittings are smaller for tunneling along $\Phi_{1}$ than along $\Phi_{2}$; this determines the energetic order of the torsional eigenstates which are shown in Figs. 3d and 3e, respectively. The wavefunction $\Psi^{\Gamma_{6}}\left(\Phi_{1}, \Phi_{2}\right)$ which is shown in Fig. 3f has two nodal lines, so that $\Psi^{\Gamma_{6}}\left(\Phi_{1}, \Phi_{2}\right)$ has the highest energy of the quadruplet of torsional wavefunctions $\Psi^{\Gamma_{1}}\left(\Phi_{1}, \Phi_{2}\right), \Psi^{\Gamma_{7}}\left(\Phi_{1}, \Phi_{2}\right), \Psi^{\Gamma_{4}}\left(\Phi_{1}, \Phi_{2}\right)$ and $\Psi^{\Gamma_{6}}\left(\Phi_{1}, \Phi_{2}\right)$ for the NSIs with nuclear spin functions having the symmetry $\Gamma_{5}, \Gamma_{3}, \Gamma_{8}$, $\Gamma_{2}$, respectively.

Finally, we identify the parity of all four torsional states. As can be seen clearly in Fig. $3 c-f$, the torsional ground state of each NSI is even under inversion. However, as our investigations show, the first excited torsional states of each NSI have odd parity. 


\section{Conclusions}

The present derivations, with exemplary demonstrations for the model CCD, show in general that molecules which have a $C_{2}$ symmetry axis and three segments $\mathrm{A}, \mathrm{B}, \mathrm{C}$ which can rotate independently about that axis (corresponding to two independent torsions, B vs. A and C vs. B, $c f$. items (i) and (ii) of the Introduction), have at most eight NSIs; for example, CCD has, indeed, eight NSIs, and four of them "survive" as $T \rightarrow 0$. Each NSI is characterized by a specific tandem of two (out of eight) irreducible representations (IREPs) for the nuclear spin and the torsional wavefunctions, provided by the character table of the subgroup $\mathrm{G}^{\mathrm{PSMS}}\left[\mathrm{G}_{16}^{\mathrm{A}}\right]$ of the molecular symmetry group $\mathrm{G}_{16}^{\mathrm{A}}$.

The model applications to CCD demonstrate the principles of the approach. It offers various possibilities for extensions, e.g. to include additional vibrational degrees of freedom ( $c f$. Ref. [67]), and/or additional electronic excited states. The molecular symmetry adapted wavefunctions with selective labels of IREPs represent different NSIs which may also serve as initial states for subsequent applications, e.g. for quantum simulations of NSI selective spectroscopy and dynamics. Work along this line is in progress. The approach may also be applied to different isotopomers, and in general to any other molecule that belongs to the class of molecules characterized by the two properties, items (i) and (ii).

\section{Acknowledgement}

We are grateful to Professors Yehuda Haas (The Hebrew University of Jerusalem) and Hans-Heinrich Limbach (Freie Universität Berlin) for stimulating discussions and continuous encouragement, and Mr. Florian Korinth for preparing Figs. 1 and 2. We thank Dr. Chris Eyles (Freie Universität Berlin) for careful reading the original version of the manuscript, and for valuable suggestions for the preparation of the final version. Financial support by the Deutsche Forschungsgemeinschaft via projects GO1059/7-3, LE 2138/2-1, and MA 515/25-3 is also gratefully acknowledged.

\section{References}

1. W. Heisenberg, Z. Physik 41 (1927) 239.

2. F. Hund, Z. Physik 42 (1927) 93.

3. T. L. Hill, An Introduction to Statistical Thermodynamics, Dover, New York (1986).

4. S. Tomonaga, The Story of Spin, The University of Chicago Press, Chicago/London (1997).

5. K. F. Bonhoeffer and P. Harteck, Z. Phys. Chem. B4 (1929) 113.

6. A. Eucken, Sitzber. Preuss. Akad. Wiss. (1912) 41.

7. R. Mecke, Z. Physik 31 (1925) 709.

8. P. R. Bunker and P. Jensen, Spectroscopy and Broken Symmetry, in: Frontiers of Molecular Spectroscopy, J. Laane (Ed.), Elsevier, Amsterdam (2009) and references cited therein.

9. M. Quack, Mol. Phys. 34 (1977) 477.

10. P. L. Chapovsky and L. J. F. Hermans, Annu. Rev. Phys. Chem. 50 (1999) 315 and references cited therein.

11. G. Buntkowsky, J. Bargon, and H.-H. Limbach, J. Am. Chem. Soc. 118 (1996) 8677.

12. G. Buntkowsky, H.-H. Limbach, F. Wehrmann, I. Sack, H. M. Vieth, and R. H. Morris, J. Phys. Chem. A 101 (1997) 4679.

13. H.-H. Limbach, S. Gründemann, G. Buntkowsky, S. Sabo-Etienne, B. Chaudret, G. J. Kubas, and J. Eckert, J. Am. Chem. Soc. 120 (1998) 7929. 
14. S. Gründemann, H.-H. Limbach, G. Buntkowsky, S. Sabo-Etienne, and B. Chaudret, J. Phys. Chem. A 103 (1999) 4752.

15. J. Matthes, T. Pery, S. Gründemann, G. Buntkowsky, S. Sabo-Etienne, B. Chaudret, and H.-H. Limbach, J. Am. Chem. Soc. 126 (2004) 8366.

16. G. Buntkowsky and H.-H. Limbach, J. Low. Temp. Phys. 143 (2006) 55.

17. G. Buntkowsky and H.-H. Limbach, Dihydrogen and symmetry: The role of symmetry on the chemistry of dihydrogen transfer in the light of NMR spectroscopy, in: Hydrogen-Transfer Reactions, Vol. 2, J. T. Hynes, J. Klinman, H.-H. Limbach, R. L. Schowen (Eds.), WileyVCH, Weinheim (2006) 639.

18. G. Buntkowsky, B. Walaszek, A. Adamczyk, Y. Xu, H.-H. Limbach, and B. Chaudret, Phys. Chem. Chem. Phys. 8 (2006) 1929.

19. H.-H. Limbach, G. Buntkowsky, J. Matthes, S. Gründemann, T. Pery, B. Walaszek, and B. Chaudret, ChemPhysChem 7 (2006) 551.

20. G. Buntkowsky, H.-H. Limbach, B. Walaszek, A. Adamczyk, Y. Xu, H. Breitzke, A. Schweitzer, T. Gutmann, M. Wächtler, J. Frydel, T. Emmler, N. Amadeu, D. Tietze, and B. Chaudret, Z. Phys. Chem. 222 (2008) 1049.

21. T. Gutmann, M. Sellin, H. Breitzke, A. Stark, and G. Buntkowsky, Phys. Chem. Chem. Phys. 11 (2009) 9170.

22. U. Bommerich, T. Trantzschel, S. Mulla-Osman, G. Buntkowsky, J. Bargon, and J. Bernarding, Phys. Chem. Chem. Phys. 12 (2010) 10309.

23. T. Gutmann, T. Ratajczyk, Y. Xu, H. Breitzke, A. Grünberg, S. Dillenberger, U. Bommerich, T. Trantzschel, J. Bernarding, and G. Buntkowsky, Solid State NMR 38 (2011) 90.

24. T. Gutmann, T. Ratajczyk, S. Dillenberger, Y. Xu, A. Grünberg, H. Breitzke, U. Bommerich, T. Trantzschel, J. Bernarding, and G. Buntkowsky, Solid State NMR 40 (2011) 88.

25. T. Ratajczyk, T. Gutmann, S. Dillenberger, S. Abdulhussaein, J. Frydel, H. Breitzke, U. Bommerich, T. Trantzschel, J. Bernarding, P. Magusin, and G. Buntkowsky, Solid State NMR 43-44 (2012) 14.

26. T. Trantzschel, J. Bernarding, M. Plaumann, D. Lego, T. Gutmann, T. Ratajczyk, S. Dillenberger, G. Buntkowsky, J. Bargon, and U. Bommerich, Phys. Chem. Chem. Phys. 14 (2012) 5601.

27. T. Trantzschel, M. Plaumann, J. Bernarding, D. Lego, T. Ratajczyk, S. Dillenberger, G. Buntkowsky, J. Bargon, and U. Bommerich, Appl. Magn. Res. 44 (2013) 267.

28. J. Matthes, S. Gründemann, G. Buntkowsky, B. Chaudret, and H.-H. Limbach, Appl. Magn. Res. 44 (2013) 247.

29. C. R. Bowers and D. P. Weitekamp, Phys. Rev. Lett. 57 (1986) 2645.

30. C. R. Bowers and D. P. Weitekamp, J. Am. Chem. Soc. 109 (1987) 5541.

31. T. C. Eisenschmid， R. U. Kirss， P. P. Deutsch，S. I. Hommeltoft, R. Eisenberg，J. Bargon, R. G. Lawler, and A. L. Balch, J. Am. Chem. Soc. 109 (1987) 8089.

32. L. S. Bouchard, S. R. Burt, M. S. Anwar, K. V. Kovtunov, I. V. Koptyug, and A. Pines, Science 319 (2008) 442.

33. J. F. Dechent, L. Buljubasich, L. M. Schreiber, H. W. Spiess, and K. Münnemann, Phys. Chem. Chem. Phys. 14 (2012) 2346.

34. S. B. Duckett and D. Blazina, Eur. J. Inorg. Chem. 2003 (2003) 2901.

35. S. B. Duckett and N. J. Wood, Coord. Chem. Rev. 252 (2008) 2278.

36. S. B. Duckett and R. E. Mewis, Acc. Chem. Res. 45 (2012) 1247

37. J. Natterer and J. Bargon, Prog. Nucl. Magn. Reson. Spectrosc. 31 (1997) 293.

38. R. A. Green, R. W. Adams, S. B. Duckett, R. E. Mewis, D. C. Williamson, and G. G. R. Green, Prog. Nucl. Magn. Reson. Spectrosc. 67 (2012) 1.

39. S. B. Ducket and C. J. Sleigh, Prog. Nucl. Magn. Reson. Spectrosc. 34 (1999) 71.

40. K. V. Kovtunov, V. V. Zhivonitko, A. Corma, and I. V. Koptyug, J. Phys. Chem. Lett. 1 (2010) 1705.

41. S. B. Ducket and N. J. Wood, Coordin. Chem. Rev. 252 (2001) 2278.

42. K. Golman, O. Axelson, H. Johannesson, S. Mansson, C. Olofsson, and J. S. Petersson, Magn. Reson. Med. 46 (2001) 1.

43. M. J. Mumma, H. A. Weaver, and H. P. Larson, Astron. Astrophys. 187 (1987) 419. 
44. S. Fleischer, I. S. Averbukh, and Y. Prior, Phys. Rev. Lett. 99 (2007) 093002.

45. S. Fleischer, I. S. Averbukh, and Y. Prior, J. Phys. B 41 (2008) 074018.

46. S. Fleischer, Y. Khodorkovsky, Y. Prior, and I. S. Averbukh, New J. Phys. 11 (2009) 105039.

47. E. Gershnabel and I. S. Averbukh, Phys. Rev. A 78 (2008) 063416.

48. T. Grohmann and M. Leibscher, J. Chem. Phys. 134 (2011) 204316.

49. T. Grohmann, Theoretische Untersuchungen zur Quantendynamik der Kernspinisomere nichtlinearer Moleküle, Dissertation, Freie Universität Berlin (2012).

50. O. Deeb, M. Leibscher, J. Manz, W. von Muellern, and T. Seideman, ChemPhysChem 8 (2007) 322.

51. T. Grohmann, O. Deeb, and M. Leibscher, Chem. Phys. 338 (2007) 252.

52. S. Belz, T. Grohmann, and M. Leibscher, J. Chem. Phys. 131 (2009) 034305.

53. S. Belz, Laser Control of Torsional Nuclear Wave Packet Dynamics Via Conical Intersections, Dissertation, Freie Universität Berlin (2011).

54. S. Al-Jabour, Molecular Symmetry, Quantum Chemistry and Dynamics: Simulation of Laser Driven Molecular Torsion in the Presence of a Conical Intersection, Dissertation, Freie Universität Berlin (2011).

55. J. Floß, T. Grohmann, M. Leibscher, and T. Seideman, J. Chem. Phys. 136 (2012) 084309.

56. J. Manz, B. Proppe, and B. Schmidt, Z. Phys. D Atom. Mol. Cl. 34 (1995) 111.

57. Y. Fujimura, L. González, K. Hoki, J. Manz, and Y. Ohtsuki, Chem. Phys. Lett. 310 (1999) 1, 578.

58. O. Brackhagen, H. Busse, J. Giraud-Girard, J. Manz, and M. Oppel, J. Chem. Phys. 112 (2000) 8819.

59. Y. Fujimura, L. González, K. Hoki, D. Kröner, J. Manz, and Y. Ohtsuki, Angew. Chem. Int. Ed. 39 (2000) 4586.

60. F. Evers, J. Giraud-Girard, S. Grimme, J. Manz, C. Monte, M. Oppel, W. Rettig, P. Saalfrank, and P. Zimmermann, in: Festschrift W. H. Miller, J. Phys. Chem. A 105 (2001) 2911.

61. K. Hoki and D. Kröner, J. Manz, Chem. Phys. 267 (2001) 59.

62. J. Manz, B. Proppe, and B. Schmidt, Phys. Chem. Chem. Phys. 4 (2002) 1876.

63. D. Kröner, Theory of Selective Preparation of Enantiomers by Laser Pulses, Dissertation, Freie Universität Berlin (2003).

64. Y. Fujimura, L. González, D. Kröner, J. Manz, I. Mehdaoui, and B. Schmidt, Chem. Phys. Lett. 386 (2004) 248.

65. D. Kröner and B. Klaumützer, Phys. Chem. Chem. Phys. 9 (2007) 5009.

66. S. Ramakrishna and T. Seideman, Phys. Rev. Lett. 99 (2007) 103001.

67. S. Alfalah, S. Belz, O. Deeb, M. Leibscher, J. Manz, and S. Zilberg, J. Chem. Phys. 130 (2009) 124318.

68. M. Barbatti, S. Belz, M. Leibscher, H. Lischka, and J. Manz, Chem. Phys. 350 (2008) 145.

69. C. B. Madsen, L. B. Madsen, S. S. Viftrup, M. P. Johansson, T. B. Poulsen, L. Holmegaard, V. Kumarappan, M. P. Jørgensen, and H. Stapelfeldt, Phys. Rev. Lett. 102 (2009) 073007.

70. C. B. Madsen, L. B. Madsen, S. S. Viftrup, M. P. Johansson, T. B. Poulsen, L. Holmegaard, V. Kumarappan, M. P. Jorgensen, and H. Stapelfeldt, J. Chem. Phys. 130 (2009) 234310.

71. S. Al-Jabour, M. Baer, O. Deeb, M. Leibscher, J. Manz, X. Xu, and S. Zilberg, J. Phys. Chem. A. 114 (2010) 2991.

72. S. Parker, M. Ratner, and T. Seideman, J. Chem. Phys. 135 (2011) 224301.

73. S. Parker, M. Ratner, and T. Seideman, Mol. Phys. 110 (2012) 1941.

74. Borden, E. T. (Ed.), Diradicals, Wiley-Interscience, New York, (1982).

75. Z. R. Grabowski, K. Rotkiewicz, and W. Rettig, Chem. Rev. 103 (2003) 3899.

76. N. M. Albu, E. Bergin, and D. J. Yaron, J. Phys. Chem. A 113 (2009) 7090.

77. S. Westenhoff, W. J. D. Beenken, R. H. Friend, N. C. Greenham, A. Yartsev, and V. Sundström, Phys. Rev. Lett. 97 (2006) 166804.

78. G. Pérez-Hernández, A. Pelzer, L. González, and T. Seideman, New. J. Phys. 12 (2010) 075007.

79. M. Shapiro and P. Brumer, Quantum Control of Molecular Processes, $2^{\text {nd }}$ edn., Wiley $-\mathrm{VCH}$, Weinheim (2012), 397. 
80. H. Ohmura and T. Nakanaga, J. Chem. Phys. 120 (2004) 5176.

81. L. Holmegaard, J. H. Nielsen, I. Nevo, H. Stapelfeldt, F. Filsinger, J. Küpper, and G. Meijer, Phys. Rev. Lett. 102 (2009) 023001.

82. F. Filsinger, J. Küpper, G. Meijer, L. Holmegaard, J. Nielsen, I. Nevo, J. L. Hansen, and H. Stapelfeldt, J. Chem. Phys. 131 (2009) 064309.

83. B. Friedrich and D. Herschbach, J. Chem. Phys. 111 (1999) 6157.

84. B. Friedrich and D. Herschbach, J. Phys. Chem. A 103 (1999) 10280.

85. C. M. Dion, A. D. Bandrauk, O. Atabek, A. Keller, H. Umeda, and Y. Fujimura, Chem. Phys. Lett. 302 (1999) 215.

86. I. Sh. Averbukh and R. Arvieu, Phys. Rev. Lett. 87 (2001) 163601.

87. M. Machholm and N. E. Henriksen, Phys. Rev. Lett. 87 (2001) 193001.

88. S. Guérin, L. P. Yatsenko, H. R. Jauslin, O. Faucher, and B. Lavorel, Phys. Rev. Lett. 88 (2002) 233601.

89. H. Sakai, S. Minemoto, H. Nanjo, H. Tanji, and T. Suzuki, Phys. Rev. Lett. 90 (2003) 083001 .

90. E. Gershnabel, I. Sh. Averbukh, and R. J. Gordon, Phys. Rev. A 73 (2006) 061401.

91. K. Nakajima, H. Abe, and Y. Ohtsuki, J. Phys. Chem. A 116 (2012) 11219.

92. H. Stapelfeldt and T. Seideman, Rev. Mod. Phys. 75 (2003) 543.

93. T. Seideman and E. Hamilton, Adv. At. Mol. Opt. Phys. 52 (2005) 282.

94. S. Belz, S. Zilberg, M. Berg, T. Grohmann, and M. Leibscher, J. Phys. Chem. A 116 (2012) 11189.

95. Cotton, F. A., Chemical Applications of Group Theory, $2^{\text {nd }}$ edn., Wiley, New York (1971).

96. H. C. Longuet-Higgins, Mol. Phys. 6 (1963) 445.

97. J. T. Hougen, J. Chem. Phys. 37 (1962) 1433.

98. J. T. Hougen, J. Chem. Phys. 39 (1963) 358.

99. P. R. Bunker and P. Jensen, Molecular Symmetry and Spectroscopy, $2^{\text {nd }}$ edn. National Research Council of Canada, Ottawa, (2002).

100. A. J. Merer and J. K. G. Watson, J. Mol. Spectrosc. 47 (1973) 499.

101. B. Lasorne, M. A. Robb, H.-D. Meyer, and F. Gatti, Chem. Phys. 377 (2010) 30.

102. R. Mirman, Group Theory: an Intuitive Approach, World Scientific, Singapore, reprint edition (2007).

103. M. Born and J. R. Oppenheimer, Ann. Phys. 84 (1927) 457.

104. E. Thiele and D. J. Wilson, J. Chem. Phys. 35 (1961) 1256.

105. B. O. Roos, P. R. Taylor, and P. E. M. Siegbahn, Chem. Phys. 48 (1980) 157.

106. T. H. Dunning Jr., J. Chem. Phys. 90 (1989) 1007.

107. M. W. Schmidt, K. K. Baldridge, J. A. Boatz， S. T. Elbert, M. S. Gordon, J. H. Jensen, S. Koseki, N. Matsunaga, K. A. Nguyen, S. Su, T. L. Windus, M. Dupuis, and J. A. Montgomery, J. Comput. Chem. 14 (1993) 1347.

108. Alex A. Granovsky, PC GAMESS version 7.1, http://classic.chem.msu.su/gran/firefly/index. html.

109. H.-J. Werner, P. J. Knowles, G. Knizia, F. R. Manby, M. Schütz, P. Celani, T. Korona, R. Lindh, A. Mitrushenkov, G. Rauhut, K. R. Shamasundar, T. B. Adler, R. D. Amos, A. Bernhardsson, A. Berning, D. L. Cooper, M. J. O. Deegan, A. J. Dobbyn, F. Eckert, E. Goll, C. Hampel, A. Hesselmann, G. Hetzer, T. Hrenar, G. Jansen, C. Köppl, Y. Liu, A. W. Lloyd, R. A. Mata, A. J. May, S. J. McNicholas, W. Meyer, M. E. Mura, A. Nicklass, D. P. O’Neill, P. Palmieri, K. Pflüger, R. Pitzer, M. Reiher, T. Shiozaki, H. Stoll, A. J. Stone, R. Tarroni, T. Thorsteinsson, M. Wang, and A. Wolf, MOLPRO, version 2010.1, a package of ab initio programs, see http://www.molpro.net.

110. C. C. Marston and G. G. Balint-Kurti, J. Chem. Phys. 91 (1989) 3571.

111. M. H. Levitt, Spin Dynamics: Basics of Nuclear Magnetic Resonance, $2^{\text {nd }}$ edn., Wiley VCH, Weinheim (2008).

112. R. McWeeny, Symmetry: an Introduction to Group Theory and its Applications, Dover, New York (2002). 